\title{
Clinical Monograph for Drug Formulary Review: Erectile Dysfunction Agents
}

\author{
HELEN ELOISE CAMPBELL, BS, PharmD
}

\begin{abstract}
BACKGROUND: Significant advances in the pharmacologic treatment of erectile dysfunction (ERD) have occurred in recent years, most notably the introduction of sildenafil, the first oral selective phosphodiesterase type 5 (PDE5) inhibitor, in 1998. Sildenafil quickly gained acceptance by the medical community and the public because of its broad efficacy for different types of ERD and its ease of use. Two PDE5 inhibitors, vardenafil and tadalafil, have since joined sildenafil to compete in the ERD market. A review was conducted by the Drug Information Service of a pharmacy benefits manager (PBM) to determine the relative merits and place in therapy of commonly used ERD drugs as part of drug formulary management process and decision making by the Pharmacy \& Therapeutics (P\&T) committee.
\end{abstract}

OBJECTIVE: To provide readers with a comprehensive clinical monograph on ERD drugs written from a managed care perspective.

METHODS: The PBM clinical monograph is designed to provide health plans with an evidence-based review of drugs, therapeutic classes, and disease states with a managed care focus. For each therapeutic class or disease review, an extensive and thorough literature search of MEDLINE is conducted for efficacy, safety, effectiveness, and humanistic and economic data. Drug/disease-state databases (UptoDate online, MICROMEDEX), U.S. Food and Drug Administration clinical reviews, key Internet sites, medical/pharmacy-related news sites, clinical guidelines, and AMCP dossiers are also reviewed. Formulary drug monographs prepared by the Drug Information Service of the PBM include a critical analysis and summary of disease-oriented and patient-oriented clinical outcomes, effectiveness, and humanistic data. Additional data considered and included in the formulary review process are clinical attributes, patent expirations/generic competition, off-label or pending indications, and pharmacoeconomic data.

RESULTS: Despite the lack of head-to-head comparative studies, all 3 PDE5 inhibitors appear to have equivalent efficacy in the treatment of general ERD and ERD associated with diabetes and postprostatectomy. Sildenafil has additional efficacy data in the management of ERD associated with spinal cord injury and antidepressant medications. Tadalafil has the longest duration of action (up to 36 hours); this feature can be both beneficial (greater sexual spontaneity) or possibly detrimental (greater exposure to drug, delayed adverse events). All 3 PDE5 inhibitors appear to be generally well tolerated and have similar contraindications and warnings. However, vardenafil is the only PDE5 inhibitor with a cardiac conduction precaution. Alprostadil products are recommended in current ERD guidelines as second-line therapy for those who have not responded or cannot take the oral PDE5 inhibitors. Overall, higher clinical efficacy rates are achieved with intracavernous than with transurethral administration.

CONCLUSION: A large amount of clinical efficacy and safety data has been published since the market launch of sildenafil in 1998. Sildenafil has the greatest body of efficacy and safety evidence. No comparative studies have been conducted with any of the PDE5 inhibitors. Differences in study populations, primary end points, and measurement tools make comparisons difficult. However, all PDE5 inhibitors appear to be roughly equivalent in efficacy, with minor differences in adverse event profiles. Until more comparative data are available, economic considerations will be a significant factor in choosing ERD products for formulary inclusion.

KEYWORDS: Erectile dysfunction, Sildenafil, Vardenafil, Tadalafil, Drug monograph, Outcomes-based formulary, Evidence-based medicine
Editors' note: This article contains the information presented in nearly identical facsimile to the Pharmacy and Therapeutics (PET) committee for the pharmacy benefit manager (PBM) and its health plan clients. Only the cost data have been updated, and the PET committee sees actual cost and utilization data for the PBM during its deliberations. Part of the purpose of this article is to present for readers an example of the information that is actually reviewed in contemporary $P E T$ processes in managed care today.

\section{Introduction}

Erectile dysfunction (ERD) has been defined as the persistent (lasting at least 6 months) inability to attain and maintain erection sufficient to permit satisfactory sexual performance. Although ERD is not a life-threatening disorder, it has a profound impact on the quality of life of those who suffer from it. ERD increases progressively with age, but it is not an inevitable consequence of aging. Other age-related conditions may increase the risk of developing ERD.

Based on the Massachusetts Male Aging Study, the probability of ERD of any degree is 40\% among 40-year-old men and 70\% among 70-year-old men.,2 Many diseases-and many medications - may lead to erectile dysfunction. Therefore, an individual evaluation and identification of the underlying causes as well as a reduction in polypharmacy and a substitution of medications should be some of the first approaches in the management of ERD. ${ }^{3}$

Significant advances in the pharmacologic treatment of ERD have occurred in recent years, most notably the introduction in 1998 of sildenafil, the first oral selective phosphodiesterase type 5 (PDE5) inhibitor. Sildenafil quickly gained acceptance by the medical community and the public because of its broad efficacy for different types of ERD and its ease of use. Recent guidelines published by the European Association of Urology and the American Association of Clinical Endocrinologists include sildenafil as first-line pharmacologic therapy in the treatment of ERD when nonspecific therapy is appropriate. ${ }^{3,4}$

\section{Author}

HELEN ELOISE CAMPBELL, BS, PharmD, is a drug information manager, WellPoint Pharmacy Management, residing in Cherry Valley, New York.

AUTHOR CORRESPONDENCE: Helen Eloise Campbell, BS, PharmD, Drug Information Manager, WellPoint Pharmacy Management, P.O. Box 9, Cherry Valley, NY 13320. Tel: (607) 264-8134; Fax (607) 264-8136;

E-mail: Helen.campbell@wellpoint.com

Copyright $\odot$ 2005, Academy of Managed Care Pharmacy. All rights reserved. 


\section{(TABLE 1$)$ Monograph Review Agents: Erectile Dysfunction}

\begin{tabular}{ll}
\hline Sildenafil & (Viagra) \\
Vardenafil & (Levitra) \\
Tadalafil & (Cialis) \\
Intracavernous alprostadil injection & (Caverject, Edex) \\
Transurethral alprostadil & (MUSE) \\
\hline
\end{tabular}

TABLE 2 Causes of Erectile Dysfunction 2,5,9

\begin{tabular}{l|l}
\hline Type of Disorder & Example \\
\hline Vascular & $\begin{array}{l}\text { Atherosclerosis, penile Raynaud's phenomenon, } \\
\text { cardiovascular disease, diabetes }\end{array}$ \\
\hline Neurological & $\begin{array}{l}\text { Spinal cord damage, cerebrovascular accident } \\
\text { Peripheral neuropathy, diabetic autonomic neuropathy }\end{array}$ \\
\hline Hormonal/endocrine & $\begin{array}{l}\text { Hypogonadism, hyperthyroidism, hyperglycemia } \\
\text { (poorly controlled diabetes) }\end{array}$ \\
\hline Psychogenic & Performance anxiety, depression \\
\hline Iatrogenic & $\begin{array}{l}\text { Pelvic radiation, lumbar sympathectomy, prostatectomy } \\
\text { Renal transplant, spinal cord resection }\end{array}$ \\
\hline Drug-induced & $\begin{array}{l}\text { Diuretics, sympatholytics, nonselective beta-blockers, } \\
\text { alpha-blockers, direct vasodilators, calcium channel } \\
\text { blockers, antidepressants, antipsychotics, anxiolytics, } \\
\text { opioids, cimetidine }\end{array}$ \\
\hline
\end{tabular}

Two PDE5 inhibitors, vardenafil and tadalafil, have joined sildenafil to compete in the ERD market. However, PDE5 inhibitors do not work for all patients, and some individuals may have contraindications that preclude their use. Other firstline options include the use of vacuum devices or investigational oral drugs such as oral yohimbine, trazodone, phentolamine, and, in Europe, sublingual apomorphine. Efficacy data is sparse and conflicting for the off-label use of trazodone, yohimbine, and phentolamine in the treatment of ERD. ${ }^{4}$

U.S. Food and Drug Administration (FDA)-approved agents recommended as second-line alternatives in ERD guidelines include intracavernosal alprostadil therapy (direct delivery of the drug to the erectile chambers) and transurethral alprostadil delivery (direct delivery to the urethra) (Table 1).

This monograph will present a short overview of the etiology, risk factors, pathophysiology, and diagnosis of ERD. The focus of this monograph will be an evaluation of pharmacology, pharmacodynamics, pharmacokinetics, clinical efficacy, and the safety of the pharmacologic treatments that are approved by the FDA for the management of ERD.

Testosterone injection, oral tablets, gels, and transdermal systems are indicated for the treatment of ERD associated with hypogonadism. The review of testosterone preparations for the treatment of hypogonadism will be the subject of a separate monograph.

\section{Overview of ERD}

\section{Pathophysiology}

Penile erection depends on one or two main mechanisms: reflex erection or psychogenic erection. It is a hemodynamic event regulated by the relaxation of the arterial and corporal smooth muscle. The penis consists of paired erection chambers (corpora cavernosa) that are filled with erectile tissue (corporal sinusoids) composed of smooth muscles. Relaxation of the smooth muscle of the corpora cavernosa is mediated by the release of acetylcholine by the parasympathetic nerves. Acetylcholine causes the endothelial cells to release a nonandrogenic, noncholinergic carrier of relaxation signal—nitric oxide. Nitric oxide may stimulate guanylate cyclase to produce cyclic guanosine monophosphate (cGMP), therefore causing a relaxation of the trabecular smooth muscle. Penile erection is a result of neurally mediated increased arterial inflow into the corporal bodies and an increased amount of oxygen that stimulate nitric oxide synthesis by cavernosal nerves and endothelium, along with a decrease or cessation of venous outflow..$^{5-7}$

The corporal smooth muscle is contracted when the penis is flaccid. The contraction is due to the presence of a normally present adrenergic tone. Smooth muscle relaxation occurs with erection. There are a number of other receptors in penile smooth muscle, including those responsive to vasoactive intestinal polypeptide, dopamine, histamine, prostaglandin, and various others. ${ }^{5-7}$

\section{Etiology and Risk Factors of ERD}

Vascular disease is the most common etiology of ERD in elderly men. The risk of vascular ERD increases with smoking, hypercholesterolemia, and diabetes. In addition, many diseases, such as diabetes, stroke, and Parkinson's disease, can cause autonomic dysfunction. This can impair the penile arterial vasodilatation, maintaining the vascular constriction, and therefore preventing erection. Furthermore, a number of medications have been associated with ERD. Medications with anticholinergic properties, such as antidepressants, antipsychotics, and antihistamines, block parasympathetic-mediated penile artery vasodilatation and trabecular smooth muscle relaxation. ${ }^{8}$ Causes contributing to ERD may be related to a number of disorders, which are listed in Table 2.

ERD is clearly a symptom of many conditions, and certain risk factors have been identified, some of which may be preventable. Diabetes mellitus, hypogonadism, hypertension, vascular disease, high cholesterol or low-density lipoprotein cholesterol, alcohol ingestion, depression, lack of sexual knowledge, poor sexual techniques, and many chronic diseases have all been identified as risk factors. In addition, age is a strong indirect risk factor because it may be associated with increased likelihood of direct risk factors. Smoking is another indirect risk factor that may increase the effects of other risk factors, such as hypertension or vascular disease. Knowledge of the risk factors can guide patients to prevention strategies. ${ }^{5,7,9,10}$ 


\section{Diagnosis of ERD}

ERD may be associated with several abnormalities of the endocrine, neurological, and vascular system. Thus, an appropriate evaluation of all men with ERD should include a medical and sexual history, physical exam, psychosocial evaluation, and appropriate laboratory studies. ${ }^{3}$

Endocrine evaluation includes hemoglobin AlC, a morning serum testosterone, prolactin, luteinizing hormone, and folliclestimulating hormone (FSH) levels. Other tests, such as complete blood count, urinalysis, creatinine, lipid profile, fasting blood sugar, and thyroid function may be indicated to exclude an unrecognized underlying systemic disease. Neurologic causes may be associated with a history of diabetes, spinal injury, or cerebrovascular accident; a detailed medical history will be essential to identify them. In addition, nocturnal penile tumescence testing may be useful when a primary psychogenic ERD is suspected. An erectile response to an intracavernosal injection of pharmacological test dose of a vasodilatory agent, such as papaverine or PGEl, indicates adequate arterial and veno-occlusive function. For patients who favor noninvasive treatments, such as the oral PDE5 inhibitors, pharmacological injection, intraurethral suppository, or vacuum constrictor devices, no further diagnostic tests are necessary. On the other hand, for patients with unsatisfactory response, penile implant surgery or further diagnostic tests may be appropriate. ${ }^{3}$

\section{Pharmacology/Pharmacodynamics \\ FDA-Approved Therapy \\ Alprostadil (Caverject, Edex, and MUSE)}

Prostaglandin El (alprostadil) is one of the prostaglandins, naturally occurring acidic lipids with a variety of pharmacological effects, including vasodilatation, inhibition of platelet aggregation, and stimulation of intestinal and uterine smooth muscle. It acts by relaxing the trabecular smooth muscles of the corpus cavernosum and increasing the diameter of cavernous arteries, and this leads to erection. In animal studies, the degree and duration of cavernous smooth muscle relaxation appears to be dose dependent. ${ }^{11-13}$

\section{PDE5 Inhibitors (Sildenafil, Vardenafil, and Tadalafil)}

The mechanism of penile erection involves relaxation of the corpus cavernosal smooth muscle. This occurs through release of nitric oxide during sexual stimulation, which results in increased concentrations of cGMP. Sildenafil, vardenafil, and tadalafil are all competitive inhibitors of the type 5 cGMPspecific PDE5 enzyme. ${ }^{14-16}$ The result is an enhancement of the effect of nitric oxide secondary to a decrease in degradation of cGMP. PDE5 inhibitors have no effect in the absence of sexual stimulation.

There are 11 families of phosphodiesterase isoenzymes that have been identified in mammalian tissue. While PDEl through 6 have been extensively studied, PDE7 through 11 have been

\begin{tabular}{|c|c|c|c|}
\hline $\begin{array}{l}\text { PDE Isoenzymes } \\
\text { Tissue Localization }\end{array}$ & Sildenafil & Vardenafil & Tadalafil \\
\hline $\begin{array}{l}\text { PDEl } \\
\text { Brain, heart, kidney, liver, skeletal muscle, } \\
\text { vascular and visceral smooth muscle }\end{array}$ & $>80$ & $>130$ & $>10,000$ \\
\hline $\begin{array}{l}\text { PDE2 } \\
\text { Adrenal cortex, brain, corpus cavernosum, } \\
\text { heart, kidney, liver, visceral smooth muscle, } \\
\text { and skeletal muscle }\end{array}$ & $>700$ & $>1,000$ & $>10,000$ \\
\hline $\begin{array}{l}\text { PDE3 } \\
\text { Corpus cavernosum, heart, platelets, } \\
\text { vascular and visceral smooth muscle, } \\
\text { liver, kidney, and adipose tissue }\end{array}$ & $>700$ & $>1,000$ & $>10,000$ \\
\hline $\begin{array}{l}\text { PDE4 } \\
\text { Kidney, lung, mast cells, brain, heart, } \\
\text { skeletal muscle, vascular and visceral } \\
\text { smooth muscle, thyroid, testis, neural tissue }\end{array}$ & $>700$ & $>1,000$ & $>10,000$ \\
\hline $\begin{array}{l}\text { PDE5 } \\
\text { Corpus cavernosum, platelets, vascular } \\
\text { and visceral smooth muscle }\end{array}$ & 1 & 1 & 1 \\
\hline $\begin{array}{l}\text { PDE6 } \\
\text { Retina }\end{array}$ & 10 & $>15$ & 700 \\
\hline $\begin{array}{l}\text { PDE7 } \\
\text { Skeletal muscle, heart, lymphocytes }\end{array}$ & $>700$ & $>1,000$ & $>10,000$ \\
\hline $\begin{array}{l}\text { PDE8 } \\
\text { Widely distributed; most abundant in } \\
\text { testes, ovaries, small intestine, and colon }\end{array}$ & $>700$ & $>1,000$ & $>9,000$ \\
\hline $\begin{array}{l}\text { PDE9 } \\
\text { Widely distributed; most abundant in } \\
\text { spleen, small intestine, and brain }\end{array}$ & $>700$ & $>1,000$ & $>9,000$ \\
\hline $\begin{array}{l}\text { PDE10 } \\
\text { Putamen and caudate nucleus, testes, thyroid }\end{array}$ & $>700$ & $>1,000$ & $>9,000$ \\
\hline $\begin{array}{l}\text { PDE11 } \\
\text { Corpus cavernosum, penile vasculature, } \\
\text { vascular smooth muscle, testes, pituitary, } \\
\text { liver, kidney, skeletal muscle }\end{array}$ & $>700$ & $>300$ & 14 \\
\hline
\end{tabular}

recently discovered, and thus less is known regarding their distribution and function in the human body.

Sildenafil, vardenafil, and tadalafil are all more selective for the PDE5 isoenzyme than for all other PDE isoenzymes. However, degrees of selectivity vary among the agents, depending on the isoenzyme in question. As illustrated in Table 3, sildenafil is 80 times more selective for PDE5 than for PDE1, but greater than 80 times more selective for PDE6, an isoenzyme heavily concentrated in the retina of the eye. ${ }^{17,18}$ In contrast, tadalafil is greater than 700 times more selective for PDE5 than for the PDE6 isoenzyme. This selectivity ratio pattern may explain why the side effect of blue-tinged vision or changes in blue-green color discrimination is reported with sildenafil but is 
TABLE 4 Hemodynamic Effects of PDE5 Inhibitors ${ }^{15,20-24}$

\begin{tabular}{|c|c|c|c|}
\hline & Sildenafil & Vardenafil & Tadalafil \\
\hline \multirow[t]{2}{*}{$\begin{array}{l}\text { Lowering of systolic and diastolic BP } \\
\text { (standing readings, hemodynamic changes are less when supine) }\end{array}$} & $\begin{array}{l}\text { Healthy volunteers } \\
\downarrow 8 \mathrm{~mm} \text { systolic } \\
\downarrow 6 \mathrm{~mm} \text { diastolic } \\
\uparrow \text { heart rate } 4 \text { BPM }\end{array}$ & $\begin{array}{l}\text { Healthy volunteers } \\
\downarrow 7 \mathrm{~mm} \text { systolic } \\
\downarrow 8 \mathrm{~mm} \text { diastolic } \\
\uparrow \text { heart rate } 4 \text { BPM }\end{array}$ & $\begin{array}{l}\text { Healthy volunteers } \\
\leftrightarrow \text { systolic } \\
\downarrow 5 \text { mm diastolic } \\
\leftrightarrow \text { heart rate }\end{array}$ \\
\hline & $\begin{array}{l}\text { No effect as compared with } \\
\text { placebo on heart rate or } \\
\text { blood pressure during } \\
\text { exercise testing in patients } \\
\text { with known or probable CAD }\end{array}$ & $\begin{array}{l}\text { CAD patients } \\
\text { NA }\end{array}$ & $\begin{array}{l}\text { CAD patients } \\
\downarrow 7 \text { mm systolic } \\
\downarrow 4 \text { mm diastolic } \\
\leftrightarrow \text { heart rate }\end{array}$ \\
\hline Effect on exercise test & $\begin{array}{l}\text { No effect on ischemic response } \\
\text { to exercise in patients with } \\
\text { known or probable CAD }\end{array}$ & $\begin{array}{l}\text { Did not affect total treadmill } \\
\text { exercise time to angina but } \\
\text { did delay onset of ST segment } \\
\text { changes in symptomatic } \\
\text { patients with stable CAD }\end{array}$ & $\begin{array}{l}\text { Did not reduce total exercise } \\
\text { or time to ischemia }\end{array}$ \\
\hline
\end{tabular}

$B P=$ blood pressure; $B P M=$ beats per minute; $C A D=$ coronary artery disease; $N A=$ not available; $P D E=$ phosphodiesterase.

not expected to occur with tadalafil use. On the other hand, tadalafil is only 14 times more selective for the PDE5 than the PDE11 isoenzyme than sildenafil and vardenafil, which have much higher selectivity ratios. The low selectivity ratio of tadalafil for PDE11, an isoenzyme heavily concentrated in the testes and skeletal muscle, led investigators to conduct safety studies to ascertain what effect tadalafil would have on spermatogenesis. However, 6-month, daily-dosing, placebocontrolled studies with 10 and $20 \mathrm{mg} /$ day of tadalafil produced no clinically relevant effect on spermatogenesis as measured by sperm count and sperm morphology and motility. Additionally, no effect was observed on hormones related to spermatogenesis (luteinizing hormone, FSH, testosterone) with chronic tadalafil use. ${ }^{19}$

\section{Hemodynamic Effect}

The PDE5 inhibitors all work as vasodilators. Because PDE5 is found in the smooth muscle of the systemic arteries and veins, these agents all have potential to interact with the cardiovascular system. Since many men with ERD also have coexisting hypertension, diabetes, and cardiovascular disease, significant hemodynamic effects from PDE5 inhibitor use could be clinically important. Table 4 summarizes the hemodynamic changes seen with the PDE5 inhibitors in normal healthy volunteers and patients with coronary artery disease. All 3 agents produce minor changes in systolic and diastolic blood pressure, but these changes do not alter response to exercise testing. Careful analysis of population data and vardenafil, sildenafil, and tadalafil clinical data do not show an increase in serious cardiac events associated with PDE5 inhibitor use. . $520-24^{-24}$

All PDE5 inhibitors are contraindicated with concomitant administration of nitrates because significant hypotension can result. Sildenafil, vardenafil, and tadalafil are also contraindicated for use with alpha-blockers for the same reason. One exception to this rule is that tadalafil can be safely administered with tamsulosin $0.4 \mathrm{mg}$ daily. ${ }^{14-16}$

\section{Effect on Cardiac Conduction}

Vardenafil in therapeutic (10 mg) and supratherapeutic (80 mg) doses produced increases in the QT interval similar to that of $400 \mathrm{mg}$ of moxafloxicin. While the clinical impact of these changes is unknown, the coadministration of vardenafil with Class IA and Class III antiarrhythmic medications should be avoided. Patients with congenital QT prolongation should also avoid vardenafil use..$^{15}$

\section{Pharmacokinetics}

The pharmacokinetics of the ERD agents are summarized in Table 5. Sildenafil and vardenafil reach peak plasma concentrations at about 1 hour after administration; tadalafil reaches peak concentrations at 2 hours. Although not well studied, efficacy data for all 3 PDE5 inhibitors indicate that onset of action is earlier (17 to 40 minutes) than when peak serum concentrations are reached. ${ }^{25-28}$ Although all 3 PDE5 inhibitors vie for the claim of earliest onset, only well-designed comparative studies will help answer the question of which agent is the fastest acting. There are no studies that directly compare the onset, duration, or overall efficacy of the PDE5 inhibitors. Unlike sildenafil and vardenafil, peak serum concentrations of tadalafil are not affected by a high-fat meal. ${ }^{14-16}$ All 3 PDE5 inhibitors undergo extensive hepatic metabolism and require some dosage adjustment with hepatic dysfunction. The most striking difference between tadalafil, vardenafil, and sildenafil is the long half-life of tadalafil (17.5 hours). This long halflife translates into a prolonged duration of action for tadalafil (up to 36 hours), earning it the name of "le weekend" drug in France. 


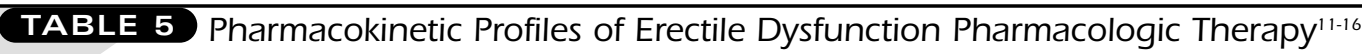

\begin{tabular}{|c|c|c|c|c|c|}
\hline & Sildenafil & Vardenafil & Tadalafil & $\begin{array}{c}\text { Alprostadil } \\
\text { Intracavernous }\end{array}$ & $\begin{array}{c}\text { Alprostadil } \\
\text { Transurethral }\end{array}$ \\
\hline Bioavailability & $40 \%$ & $15 \%$ & Not determined & $98 \%$ & $7 \%-23 \%$ \\
\hline Time to maximum concentrations ( $\mathrm{Cmax}$ ) & 1 hour & 1 hour & 2 hours & $2-5$ minutes & 16 minutes \\
\hline Change in Tmax with food & Decrease $29 \%$ & Decrease $18 \%-50 \%$ & No effect & Not applicable & Not applicable \\
\hline Volume of distribution & $105 \mathrm{~L}$ & $208 \mathrm{~L}$ & $63 \mathrm{~L}$ & Not determined & Not determined \\
\hline Metabolism & $\begin{array}{l}\text { Hepatic metabolism } \\
\text { CYP 3A4 (major) } \\
\text { CYP2C9 (minor) } \\
\text { active metabolite }\end{array}$ & $\begin{array}{l}\text { Hepatic metabolism } \\
\text { CYP 3A4 (major) } \\
\text { CYP2C and } \\
\text { CYP2A (minor) } \\
\text { active metabolite }\end{array}$ & $\begin{array}{c}\text { Hepatic metabolism } \\
\text { CYP 3A4 (major) } \\
\text { inactive metabolite }\end{array}$ & $\begin{array}{l}\text { Oxidation and } \\
\text { reduction to } \\
\text { active metabolites }\end{array}$ & $\begin{array}{l}\text { Oxidation and } \\
\text { reduction to } \\
\text { active metabolites }\end{array}$ \\
\hline Half-life (tl/2) & 4 hours & 4 hours & 17.5 hours & 8 minutes & 1-10 minutes \\
\hline \% elimination feces/urine & $80 / 13$ & $92 / 5$ & $61 / 31$ & $88 / 12$ & $90 / 10$ \\
\hline
\end{tabular}

$C Y P=$ cytochrome $P 450$ isoenzyme; Cmax=maximum concentration.

The bioavailability of intracavernous administration of alprostadil has not been studied. The absorption with transurethral administration of alprostadil appears to be biphasic, with $80 \%$ of the dose being absorbed within 10 minutes. ${ }^{11-13}$ The onset of action after intracavernous injection is within 2 to 5 minutes of administration. The onset of action after transurethral administration is slower, at about 5 to 10 minutes. Following intracavernous and transurethral administration of alprostadil, the drug is either metabolized locally or cleared from the penis into the systemic circulation and then metabolized by the lungs. The mean peripheral plasma concentrations are not significantly greater than baseline levels of endogenous alprostadil. The metabolites are excreted primarily by the kidney. Within 24 hours following administration, about $90 \%$ of the dose was excreted in urine, and the remaining 10\% was excreted in feces. The effect of age, gender, and renal or hepatic failure on the pharmacokinetics of alprostadil has not been evaluated. However, patients with pulmonary disease may have reduced ability to clear the drug because of pulmonary first-pass metabolism of prostaglandin E1. ${ }^{11-13,29}$

\section{Clinical Trials}

\section{Table Organization}

The clinical efficacy of the PDE5 inhibitors and intracavernous and transurethral alprostadil are summarized in Tables 6 through 9. The tables are organized in the following manner:

- Pivotal placebo-controlled trials in the general ERD population (Table 6)

- Pivotal placebo-controlled studies in special populations: subjects with ERD associated with diabetes, postprostatectomy, spinal cord injury, depression, and antidepressant use (Table 7)

- Comparative clinical studies (Table 8)

- Efficacy in ERD patients who have failed previous drug therapy (Table 9)

\section{Considerations in the Interpretation of ERD Drug Trials}

There are no head-to-head studies comparing the efficacy of one PDE5 inhibitor with another. While it may be tempting to compare the efficacy results seen with tadalafil and vardenafil with sildenafil, this practice is fraught with error since studies may have differing designs, study populations (age, ERD etiology, ERD severity, comorbidity, prior ERD drug use), and outcomes measures. ${ }^{30}$

\section{Outcomes Measures Used in ERD Drug Trials}

There are several primary and secondary efficacy measures commonly used in ERD clinical studies. The most common included the International Index of Erectile Function (IIEF), Sexual Health Inventory for Men (SHIM), Sexual Encounter Profile (SEP) Diary, and global assessment questions. A brief definition of each measurement tool is provided. ${ }^{30}$

\section{International Index of Erectile Function (IIEF)}

The IIEF is a validated self-administered questionnaire used to assess therapeutic efficacy of ERD therapy. It is comprosed of 5 domains:

1. Erectile function (Questions 1-5 and 15, total maximum score of 30; score of $26=$ normal erectile function; $22-25=$ mild ERD; 17-21=mild-to-moderate ERD; 11-16=moderate ERD; and $1-10=$ severe ERD). Of the ERD domain questions, 2 questions are often isolated as separate outcomes measures. The questions are: "When you attempted intercourse, how often were you able to penetrate your partner?" (IIEF question 3) and "During sexual intercourse, how often were you able to maintain your erections?" (IIEF question 4).

2. Libido

3. Orgasmic function

4. Sexual satisfaction

5. Overall satisfaction

IIEF outcomes may be reported in a variety of ways: change 


\section{TABLE 6 Erectile Dysfunction Placebo-Controlled Studies: General Population}

\section{Fink $^{31}$ (2002) Sildenafil vs. Placebo \\ $\mathrm{N}=3,229$}

Design and baseline characteristics:

Systematic review of R, PC studies of sildenafil in ERD of various etiologies

Patient age: mean age 55 years, $21 \% 65$ years or older

Ethnicity: white 70\%, Asian 21\%, African American 5\%

Comorbid conditions: HTN 28\%, ischemic heart disease $10 \%$, prostatectomy $4 \%$, diabetes $22 \%$

ERD type (all patients): organic $70 \%$, psychogenic $11 \%$, mixed $18 \%$

Duration: 4.7 years

Severity (all patients): none 2\%-3\%, mild-moderate $44 \%$, severe $47 \%$

\section{Drug regimen and duration:}

Flexible-dose design: 14 studies included, $\mathrm{N}=2,283$

Sildenafil $25 \mathrm{mg}-100 \mathrm{mg}$ or placebo x 12 weeks

Fixed-dose design: 2 studies, $\mathrm{N}=946$

Sildenafil $25 \mathrm{mg}, 50 \mathrm{mg}$, or $100 \mathrm{mg}$ or placebo x 12 weeks

Outcomes measures: IIEF Q.3 (penetration) and Q.4 (maintenance of erection), mean \% successful intercourse, global efficacy Q.1

\section{Results:}

Flexible-dose design: sildenafil vs. placebo

Mean \% successful intercourse: $57 \%$ vs. 21\%, WMD 34\% (CI, 29-38)

\% improvement in erection: $78 \%$ vs. $25 \%$, RBI 3.1 (CI, 2.7-3.5)

IIEF Q.3 scores: 3.8 vs. 2.3; WMD 1.4; CI, 1.3-1.5

IIEF Q.4 scores: 3.6 vs. 2.1; WMD 1.5; CI, 1.4-1.6

Fixed-dose design: sildenafil vs. placebo

Mean \% successful intercourse: 25 mg (43\% vs. 17\%; WMD 26; CI, 18-35) $50 \mathrm{mg}$ (50\% vs. $14 \%$; WMD 36; CI, 30-42) $100 \mathrm{mg}$ (51\% vs. 14\%; WMD 36; CI, 31-42)

\% improvement in erections: $25 \mathrm{mg}$ (66\% vs. $29 \%$; WMD 2.2; CI, 1.9-2.6) $50 \mathrm{mg}$ (76\% vs. $27 \%$; WMD $2.8 ; \mathrm{CI}, 2.3-3.4)$ $100 \mathrm{mg}(82 \%$ vs. $25 \%$; WMD 3.2; CI, 2.7-3.8)

\section{Comments:}

- Additional analyses were conducted to assess efficacy in the following subgroups: age 65 years, Asians, African Americans, severity of ERD, HTN, vascular disease, diabetes, depression, or psychogenic ERD, history of radical prostatectomy, and spinal cord disorders. While degree of efficacy varied among subgroups, all sildenafil participants had significantly higher efficacy measures than the respective placebo groups.

- $48 \%$ of the men on sildenafil had at least 1 adverse event compared with $36 \%$ of men on placebo (RRI 1.4, CI 1.3-1.6)

- Most common events were headache (11\%), flushing (12\%), dyspepsia (5\%), and visual disturbances (3\%).

- Differences in angina or cardiac chest pain did not reach statistical significance nor did rates of myocardial infarction or death.

Hellstrom $^{32}$ (2002) $\quad$ Vardenafil vs. Placebo $\quad \mathrm{N}=805$

Design and baseline characteristics:

MC, R, PC, 4-arm, parallel group, fixed-dose study

Mean age: 57 years

Etiology of ERD: organic 59\%, psychogenic 8\%, mixed 33\%

Duration: 6 years

Comorbidities: HTN 37\%, diabetes 18\%

Prior sildenafil use: $71 \%$, no sildenafil failures

\section{Drug regimen and duration:}

Vardenafil $5 \mathrm{mg}(\mathrm{N}=205), 10 \mathrm{mg}(\mathrm{N}=206), 20 \mathrm{mg}(\mathrm{N}=197)$; placebo $(\mathrm{N}=197)$

Duration: 26 weeks

Outcomes measures: IIEF erectile function domain Q.3 (penetration) and Q.4 (maintenance of erection), global efficacy Q.1

\section{Results:}

- All dosage levels of vardenafil significantly improved IIEF scores and global efficacy as compared with placebo.

- $\%$ vaginal penetration: vardenafil $65 \%-80 \%$ vs. placebo $52 \%, P<0.001$

- \% maintenance of erection: vardenafil $50 \%-65 \%$ vs. placebo $32 \%, P<0.001$

- \% improvement in erections: vardenafil $65 \%-85 \%$ vs. placebo $28 \%, P<0.001$

Comments:

- 30\%-45\% of patients in each treatment group had severe ERD at baseline.

- Efficacy increased with increasing vardenafil dose.

\begin{tabular}{ccc}
\hline Porst $^{33}(2003)$ & Vardenafil vs. Placebo & $\mathrm{N}=580$ \\
\hline \multicolumn{3}{c}{ Design and baseline characteristics: } \\
\hline
\end{tabular}

MC, R, PC, fixed-dose study

Mean age: 52 years

Etiology of ERD: organic 27\%-33\%, psychogenic 25\%-30\%, mixed 36\%-48\% Baseline ERD severity: mild 26\%-28\%, moderate 34\%-37\%, severe 32\%-36\% Prior sildenafil use: $50 \%$

\section{Drug regimen and duration:}

Vardenafil $5 \mathrm{mg}(\mathrm{N}=146), 10 \mathrm{mg}(\mathrm{N}=140), 20 \mathrm{mg}(\mathrm{N}=147)$; placebo $(\mathrm{N}=147)$ Duration: 12 weeks

Outcomes measures: IIEF erectile function domain Q.3 (penetration) and Q.4 (maintenance of erection); IIEF intercourse satisfaction, orgasmic and overall satisfaction domains

Results: Vardenafil significantly improved all IIEF domain scores vs. placebo $(P<0.010)$, no statistically significant differences in efficacy were seen at different vardenafil doses, vardenafil improvements in efficacy were not influenced by subject age, ERD type or severity, or past sildenafil use.

Comments: Headache, blurry vision, and dyspepsia were the most common adverse events.

Brock $^{34}$ (2002) Tadalafil vs. Placebo $\quad \mathrm{N}=1,112$

Design and baseline characteristics:

Pooled analysis of $5 \mathrm{MC}, \mathrm{R}, \mathrm{DB}, \mathrm{PC}$, parallel group fixed-dose studies

Mean age: 59 years; $30 \%>65$ years

ERD type: organic $61 \%$, psychogenic $9 \%$, mixed $31 \%$

Baseline ERD severity: mild 41\%, moderate 23\%, severe 36\%

Comorbidities: HTN $30 \%$, coronary artery disease $8 \%$, diabetes $21 \%$, depression 5\%

Drug regimen and duration:

Tadalafil $2.5 \mathrm{mg}(\mathrm{N}=74), 5 \mathrm{mg}(\mathrm{N}=151), 10 \mathrm{mg}(\mathrm{N}=321), 20 \mathrm{mg}$ $(\mathrm{N}=258)$; placebo $(\mathrm{N}=308)$

Duration: 12 weeks

Outcomes measures: IIEF all domains, SEP Q.2 ("Were you able to insert your penis in your partner's vagina?"), SEP Q.3 (Did your erection last long enough for you to have successful intercourse?"), global efficacy Q.1

Results:

Tadalafil vs. placebo

IIEF erectile function domain score (change from baseline)

$2.5 \mathrm{mg}(3.2$ vs. $0.6, P<0.05)$

$5.0 \mathrm{mg}(4.6$ vs. $0.6, P<0.001)$

$10 \mathrm{mg}(6.5$ vs. $0.6, P<0.001)$

$20 \mathrm{mg}(7.9$ vs. $0.6, P<0.001)$

SEP Q.2

$2.5 \mathrm{mg}(56 \%$ vs. $48 \%, P<0.001)$

$5.0 \mathrm{mg}(57 \%$ vs. $48 \%, P<0.001)$

$10 \mathrm{mg}(73 \%$ vs. $48 \%, P<0.001)$

$20 \mathrm{mg}(80 \%$ vs. $48 \%, P<0.001)$
SEP Q.3

$2.5 \mathrm{mg}(36 \%$ vs. $32 \%, P<0.05)$

$5.0 \mathrm{mg}(40 \%$ vs. $32 \%, P<0.001)$

$10 \mathrm{mg}(58 \%$ vs. $32 \%, P<0.001$ )

$20 \mathrm{mg}(75 \%$ vs. $32 \%, P<0.001)$

$\%$ successful intercourse attempts Tadalafil 2.5 mg-20 mg 36\%-75\% vs. placebo $32 \%$ 


\section{TABLE 6 Erectile Dysfunction Placebo-Controlled Studies: General Population (continued)}

Comments:

- Compared with placebo, tadalafil significantly improved all efficacy outcomes.

- High placebo response rate reflects higher proportion of subjects with mild ERD at study entry.

Linet $^{36}$ (1993) Intracavernous Alprostadil vs. Placebo $\quad \mathrm{N}=296$

Design and baseline characteristics:

MC, DB, R, parallel design, fixed-dose study

Mean age: 54 years

\section{Drug regimen and duration:}

Caverject (intracavernous alprostadil) $2.5 \mathrm{mcg}(\mathrm{N}=57), 5.0 \mathrm{mcg}(\mathrm{N}=60)$,

$10 \mathrm{mcg}(\mathrm{N}=62), 20 \mathrm{mcg}(\mathrm{N}=58)$; placebo $(\mathrm{N}=59)$

Single-dose study

Outcomes measures: clinical evaluation of erection quality, RigiScan

evaluation of erection quality

\section{Results:}

No response to placebo by either clinical or RigiScan evaluation. Men

responding with full erection ranged from roughly $20 \%$ (2.5 mcg dose)

to $50 \%$ (20 mcg dose) by either clinical or RigiScan assessment.

\section{Comments:}

- Prolonged erections occurred in 5 men; in 2 men, the erections lasted 4 hours or more.

- Mean duration of erection was related to dose.

- Penile pain was reported by $23 \%$ of the men on intracavernous alprostadil.

Albrecht abstract $^{37}$ (1997) $\quad$ Intracavernous Alprostadil vs. Placebo $\quad \mathrm{N}=233$

Design:

PC, DB, MC, crossover study

\section{Drug regimen:}

In-office dose titration phase: Study $1(\mathrm{~N}=85)$

Edex (intracavernous alprostadil) $1 \mathrm{mcg}-20 \mathrm{mcg}$ or placebo

Home phase: Study $2(\mathrm{~N}=158)$

Intracavernous alprostadil $1 \mathrm{mcg}-40 \mathrm{mcg}$ or placebo

Study 1 responders continued with the home phase; patients continued on optimal dose for 1 week then crossed over to alternate treatment.

Outcomes measures: erection adequate for successful intercourse (physician and patient assessments)

Results:

Study 2. Home phase

- \% adequate erections: intracavernous alprostadil $73 \%-74 \%$ vs. placebo $7 \%-13 \%$

- Median time to erection: intracavernous alprostadil 10 minutes

- Median duration of erection: intracavernous alprostadil 59 minutes

Comments:

- Average intracavernous alprostadil dose not reported; little information provided in this abstract.
- Prolonged erection (4-6 hours): intracavernous alprostadil 3\% vs. placebo $0.4 \%$

- Bleeding: intracavernous alprostadil 6\% vs. placebo $3 \%$

- Pain: intracavernous alprostadil 31\% vs. placebo $9 \%$

Hellstrom $^{38}$ (1996) $\quad$ Transurethral Alprostadil vs. Placebo $\quad \mathrm{N}=68$

Design:

MC, DB, PC study

Mean age: 58.6 years

Most subjects had ERD of organic etiology

Drug regimen:

MUSE (transurethral alprostadil) $125 \mathrm{mcg}, 250 \mathrm{mcg}, 500 \mathrm{mcg}$, or 1,000 mcg; placebo Duration: 2-4 weeks

Outcomes measures: erection assessment scale, \% attainment full erection,

$\%$ adequate erection for intercourse

Results:

$75.4 \%$ of alprostadil patients attained full erection on at least 1 occasion vs. $12.7 \%$ on placebo.

$49 \%$ of alprostadil patients achieved adequate erection for intercourse on at least 1 occasion.

Comments:

- Penile pain occurred in 9\%-18\% of patients.

- No reports of priapism.

Padma-Nathan $^{39}$ (1997) $\quad$ Transurethral Alprostadil vs. Placebo $\quad \mathrm{N}=1,511$

Design:

MC, DB, PC study

Mean age: 61 years

ERD of various etiologies

\section{Drug regimen:}

In-office titration to response

MUSE (transurethral alprostadil) $125 \mathrm{mcg}-1,000 \mathrm{mcg}$

3 month, at-home phase (transurethral alprostadil responders)

Transurethral alprostadil $(\mathrm{N}=485)$, placebo $(\mathrm{N}=511)$

Outcomes measures: erection assessment scale (score 4 or 5 considered a response), patient diary, \% patients with at least 1 successful intercourse

Results:

- In-clinic phase: $66 \%$ of men had at least 1 erection adequate for intercourse.

- At-home phase: transurethral alprostadil vs. placebo; erections resulting in intercourse: $65 \%$ vs. $19 \%, P<0.001$

Comments:

- Efficacy was similar regardless of age or ERD etiology.

- $11 \%$ of subjects reported mild penile pain.

- No reports of priapism.

- Hypotension occurred in 3\% of alprostadil treated patients.

$C I=$ confidence interval; $D B=$ double blind; ERD=erectile dysfunction; HTN=hypertension; IIEF=International Index of Erectile Function; MC = multicenter; $M U S E=$ Medicated Urethral System for Erection; $P C=$ placebo controlled; $Q=$ question; $R=$ randomized; $R B I=$ relative benefit increase; $R R I=$ relative risk increase; $S E P=$ Sexual Encounter Profile; WMD=weighted mean difference.

IIEF Question 3 or SEP Question 2: "When you attempted intercourse how often were you able to penetrate your partner?" IIEF Question 4 or SEP Question 3: "During sexual intercourse, how often were you able to maintain your erection after you had penetrated your partner?"; global efficacy question 1: "Did treatment improve your erections"; global efficacy question 2: "Did treatment improve your ability to have sexual intercourse?" 


\section{TABLE 7 Erectile Dysfunction Placebo-Controlled Studies: Special Populations}

Rendell $^{40}$ (1999) Sildenafil vs. Placebo in Diabetic Population $\quad N=268$

Design and baseline characteristics:

MC, R, DB, PC, flexible-dose-escalation study

Patient age: mean age 55 years; $21 \% \geq 65$ years

Comorbid conditions: type 1 diabetes 19\%, type 2 diabetes 81\%, HTN 53\%, ischemic heart disease $26 \%$

ERD type (all patients): organic 96\%, mixed 4\%

Duration: $5.3-5.8$ years

\section{Drug regimen and duration:}

Sildenafil $(\mathrm{N}=136)$

$25 \mathrm{mg}-100 \mathrm{mg}$ as needed but no more than once daily

Placebo $(\mathrm{N}=132)$

Duration: 12 weeks

Outcomes measures: mean \% successful intercourse, global efficacy (improvement in erections), IIEF score Q.3, IIEF score Q.4; IIEF erectile domain

\section{Results:}

Sildenafil vs. placebo

Mean \% successful intercourse $48 \%$ vs. $12 \%, P<0.001$

$\%$ improvement in erections

IIEF Q.3 scores

3.2 vs. $2.0, P<0.001$

IIEF Q.4 scores

$56 \%$ vs. $10 \%, P<0.001$

2.9 vs. $1.6, P<0.001$

\section{Comments:}

- In an analysis of subgroups, sildenafil efficacy was not affected by age, duration of ERD, or the duration of diabetes.

- Common adverse events included headache, dyspepsia, and respiratory tract disorder (sinus congestion or drainage).

- No discontinuations due to adverse events

Boulton $^{41}$ (2001) Sildenafil vs. Placebo in Diabetic Population $\quad \mathrm{N}=219$

Design and baseline characteristics:

MC, R, DB, PC study

Mean age: 59 years

Comorbidities: type 2 diabetes

\section{Drug regimen and duration:}

Sildenafil $(\mathrm{N}=110)$

$25 \mathrm{mg}-100 \mathrm{mg}$ as needed but no more than once daily

Placebo $(\mathrm{N}=109)$

Duration: 12 weeks

Outcomes measures: global efficacy (improvement in erections), IIEF score

Q.3, IIEF score Q.4, IIEF erectile domain

\section{Results:}

Sildenafil vs. placebo

$\%$ improvement in erections $65 \%$ vs. $11 \%, P<0.0001$

$$
\begin{aligned}
& \text { IIEF Q. } 3 \text { scores } \\
& 3.42 \text { vs. } 1.86, P<0.0001 \\
& \text { IIEF Q.4 scores } \\
& 3.35 \text { vs. } 1.84, P<0.0001
\end{aligned}
$$

Comments: Results are very similar to those attained earlier by Rendell ${ }^{39}$ (1999).

Stuckey $^{42}$ (2003) Sildenafil vs. Placebo in DiabeticPopulation $\quad N=188$

Design and baseline characteristics:

MC, R, DB, PC, flexible-dose-escalation study

Mean age: 48 years

Etiology of ERD: type 1 diabetes

Comorbidities: HTN 32\%, cardiovascular disease 36\%

Drug regimen and duration:

Sildenafil $(\mathrm{N}=95)$

$25 \mathrm{mg}-100 \mathrm{mg}$ as needed but no more than once daily

Placebo $(\mathrm{N}=93)$

Duration: 12 weeks

Outcomes measures: global efficacy (improvement in erections), IIEF score Q.3, IIEF score Q.4, IIEF erectile domain

Results:

Sildenafil vs. placebo

$\%$ improvement in erections

Mild-moderate ERD: $66 \%$ vs. $29 \%$;

Severe ERD: $30 \%$ vs. $10 \%$

IIEF Q.3 scores

3.61 vs. $2.71, P<0.001$

IIEF Q.4 scores

3.25 vs. $2.19, P<0.001$

Comments: Overall, men with mild-to-moderate ERD at baseline had higher scores for all efficacy measures than those participants with severe disease.

Goldstein $^{43}$ (2003) Vardenafil vs. Placebo in the Diabetic Population $\quad N=430$

Design and baseline characteristics:

MC, R, PC, parallel group, fixed-dose study

Mean age: 57 years

ERD type: type 1 and type 2 diabetes

Baseline ERD severity: severe 56\%, moderate 23\%, mild-moderate 15\%, mild 6\%

Comorbidities: HTN 53\%, depression 10\%

Prior sildenafil use: $58 \%$

Drug regimen and duration:

Vardenafil $10 \mathrm{mg}(\mathrm{N}=149)$

Vardenafil $20 \mathrm{mg}(\mathrm{N}=141)$

Placebo $(\mathrm{N}=140)$

Duration: 12 weeks

Outcomes measures: IIEF erectile function domain scores, SEP Q.2

(penetration), SEP Q.3 (successful intercourse), global efficacy

(improvement in erections)

\section{Results:}

Vardenafil vs. placebo

Improvement in IIEF scores

5.9-10.8 vs. $1.4 ; P<0.0001$

SEP Q.2

$$
61 \%-64 \% \text { vs. } 36 \% ; P<0.0001
$$

SEP Q.3
49\%-54\% vs. $23 \% ; P<0.0001$
$\%$ improvement in erections
57\%-72\% vs. $13 \% ; P<0.0001$

Comments

- Efficacy was usually greater for vardenafil $20 \mathrm{mg}$ than with vardenafil $10 \mathrm{mg}$

- Both dosage levels of vardenafil were statistically superior to placebo in improving IIEF scores, successful intercourse, and improvement in erections.

- Significant treatment response occurred regardless of ERD severity.

- Adverse events included headache, flushing, rhinitis, and transient vision changes (haziness).

DeTajada $^{44}$ (2002) Tadalafil vs. Placebo in the Diabetic Population $\quad \mathrm{N}=216$

Design and baseline characteristics:

MC, R, PC, parallel group, fixed-dose study

Mean age: 56 years

ERD severity: severe $72 \%$

Comorbities: HTN 37\%, hypercholesterolemia 18\%

Drug regimen and duration:

Tadalafil $10 \mathrm{mg}(\mathrm{N}=73)$

Tadalafil $20 \mathrm{mg}(\mathrm{N}=72)$

Placebo $(\mathrm{N}=71)$

Duration: 12 weeks 
TABLE 7 Erectile Dysfunction Placebo-Controlled Studies: Special Populations (continued)

Outcomes measures: IIEF erectile domain, SEP Q.2 (penetration), SEP Q.3 (successful intercourse), global efficacy (improvement in erections and improvement in sexual activity)

\section{Results:}

Tadalafil vs. placebo

Improvement in IIEF score

6.4-7.3 vs. $0.1, P<0.001$

SEP Q.2

$22 \%$ vs. no response, $P<0.001$

\author{
SEP Q.3 \\ $28-29 \%$ vs. $1.9 \%, P<0.001$ \\ Improvement in erections and \\ sexual activity \\ $56 \%-64 \%$ vs. $25 \%, P<0.001$
}

Comments: Response rates are lower in this study; however, $>72 \%$ of subjects had severe ERD by IIEF scores at study entry.

\section{Zippe $^{45}$ (2000) Sildenafil in Postprostatectomy ERD $\quad \mathrm{N}=91$}

Design and baseline characteristics:

Open-label, retrospective study

Mean age: 62 years

ERD etiology: postradical prostatectomy

Prostatectomy types: bilateral nerve sparing 58\%, unilateral nerve sparing 13\%, non-nerve sparing 29\%

Drug regimen and duration:

Sildenafil $50 \mathrm{mg}-100 \mathrm{mg} \quad(\mathrm{N}=91)$

Trial of 6-8 tablets

Outcomes measures: IIEF erectile domain, IIEF Q.3, Q.4, Cleveland Clinic postprostatectomy questionnaire

Results:

IIEF responders: bilateral nerve sparing $72 \%$, unilateral nerve sparing $50 \%$, non-nerve sparing $15 \%$

Comments:

- Patients took an average of 6-8 doses of sildenafil.

- Higher response rates with bilateral nerve-sparing procedures

\begin{tabular}{lll}
\hline Raina $^{46}$ (2003) & Sildenafil in Postprostatectomy ERD & $\mathrm{N}=48$ \\
\hline
\end{tabular}

Design:

Open-label, retrospective, 3-year follow-up of sildenafil responders from the Zippe $^{44}$ (2000) study

Mean age: 62 years

ERD etiology: postprostatectomy

Prostatectomy types: bilateral nerve sparing 58\%, unilateral nerve sparing 13\%, non-nerve sparing 29\%

Drug regimen and duration:

Sildenafil $50 \mathrm{mg}-100 \mathrm{mg} \quad(\mathrm{N}=48)$

Trial of 6-8 tablets

Outcomes measures: SHIM (measures erectile functioning)

\section{Results:}

At 3 years, $71 \%$ of original sildenafil responders were still responders. Of the

$71 \%$ responders, $31 \%$ increased the sildenafil dose from $50 \mathrm{mg}$ to $100 \mathrm{mg}$.

Comments:

- Drop-out rate was 27\%.

- Half of the discontinuations were from return of natural erections, 5 from loss of efficacy, and 1 from death of spouse.

\begin{tabular}{l}
\hline Zagaja $^{47}(2000) \quad$ Sildenafil in Postprostatectomy ERD $\quad \mathrm{N}=120$ \\
\hline Design: \\
Open-label, retrospective study \\
Age: $<55$ years $23 \%, 56-65$ years $54 \%,>65$ years $23 \%$ \\
ERD etiology: postprostatectomy \\
Prostatectomy types: bilateral nerve sparing $49 \%$, unilateral nerve sparing \\
$34 \%$, non-nerve sparing $17 \%$
\end{tabular}

Drug regimen:

Sildenafil 50 mg-100 mg ( $\mathrm{N}=120)$

Outcomes measures: 13-question survey designed to determine preoperative and postoperative erectile function, response to sildenafil and side effects

Results:

Response rates by age

Bilateral nerve sparing

$\begin{array}{cc}\text { Age }<55 \text { years } & 80 \% \\ 56-65 \text { years } & 45 \% \\ >66 \text { years } & 33 \%\end{array}$

Non-nerve sparing: no response

Unilateral nerve sparing

Age $<55$ years $\quad 40 \%$

$56-65$ years $0 \%$

$>66$ years $\quad 0 \%$

Comments: Highest response rates with younger age and bilateral nerve-

sparing procedure

Brock $^{48}$ (2003) Vardenafil vs. Placebo in Postprostatectomy ERD $\quad \mathrm{N}=440$

Design and baseline characteristics:

R, DB, PC, parallel group, fixed-dose study

Patient age: 60 years

ERD type: postprostatectomy, $73 \%$ had bilateral nerve-sparing procedures

ERD severity: severe $67 \%-74 \%$, moderate $12 \%-19 \%$, mild-moderate $11 \%-14 \%$

Comorbidities: HTN 29\%-32\%, hypercholesterolemia 21\%, depression 1\%-7\%,

past smoker $46 \%-55 \%$

Prior sildenafil use: $80 \%$

Drug regimen and duration:

Vardenafil $10 \mathrm{mg}(\mathrm{N}=146)$

Vardenafil $20 \mathrm{mg}(\mathrm{N}=149)$

Placebo $(\mathrm{N}=145)$

Duration: 12 weeks

Outcomes measures: IIEF erectile function domain scores, SEP Q.2 (pene-

tration), SEP Q.3 (successful intercourse), global efficacy (improvement in erections)

\section{Results:}

Vardenafil vs. placebo

Global efficacy-improvement Improvement in IIEF scores

in erection

$60 \%-65 \%$ vs. $13 \%, P<.0001$

Improvement in SEP Q.3 by

baseline ERD severity

Mild-moderate $70 \%-74 \%$ vs. $48 \%$

Moderate $52 \%-67 \%$ vs. $19 \%$

Severe $24 \%-28 \%$ vs. $4 \%$

Comments: Patients with mild ERD at study entry had the highest response rates.

Data on file, Eli Lilly and Company ${ }^{49}(2003)$

Tadalafil vs. Placebo in Postprostatectomy ERD

$\mathrm{N}=303$

Design and baseline characteristics:

MC, R, DB, PC, parallel group, fixed-dose study

Mean age: 60 years

Etiology of ERD: bilateral nerve-sparing prostatectomy

ERD severity: severe ERD 63\%

\section{Drug regimen and duration:}

Tadalafil $20 \mathrm{mg}(\mathrm{N}=201)$

Placebo $(\mathrm{N}=102)$

Duration: 12 weeks

Outcomes measures: IIEF erectile domain, SEP Q.2 (penetration), SEP Q.3

(successful intercourse), global efficacy (improvement in erections) 


\section{TABLE 7 Erectile Dysfunction Placebo-Controlled Studies: Special Populations (continued)}

Results:

Tadalafil vs. placebo

Improvement in IIEF

5.3 vs. $1.1, P<0.001$
SEP Q.2

$54 \%$ vs. $32 \%, P<0.001$

SEP Q.3

$41 \%$ vs. $19 \%, P<0.001$

Comments:

- All patients had bilateral nerve-sparing procedures, which are associated with a higher treatment success rate than unilateral or non-nerve-sparing procedures.

- Adverse events included headache, dyspepsia, myalgia, back pain, nasal congestion, flushing, and fatigue.

\section{Giuliano $^{50}$ (1999)}

Sildenafil vs. Placebo in the Spinal Cord Injury Population

$$
\mathrm{N}=178
$$

Design and baseline characteristics:

R, DB, PC, 2-way crossover, flexible-dose-escalating study

Mean age: 38 years

Etiology of ERD: post-SCI

\section{Drug regimen and duration:}

Sildenafil $50 \mathrm{mg}-100 \mathrm{mg}$ or placebo for 6 weeks then crossover to placebo or sildenafil for an additional 6 weeks

Duration: 6 weeks on each treatment

Median 8.5 doses of sildenafil

Outcomes measures: global efficacy question (improvement of erections),

$\%$ of successful intercourse attempts, IIEF erectile function domain Q.3 (penetration) and Q.4 (erection maintenance)

\section{Results:}

Sildenafil vs. placebo

Improvement of erections

$78 \%$ vs. $4 \%, P<0.0001$

\section{$\%$ of successful intercourse attempts $55 \%$ vs. $0 \%, P<0.001$ \\ Significant improvement in scores for IIEF Q.3 and Q.4 for sildenafil vs. placebo}

\section{Comments:}

- Most common adverse events were headache, facial flushing, nasal congestion, dyspepsia, and visual disturbances

- Significant improvement persisted even when patients with no residual erectile function at baseline were included.

- Response to sildenafil for subjects with SCI is comparable to response seen in ERD subjects with other comorbid conditions.

\section{Seidman ${ }^{51}$ (2001) \\ Sildenafil vs. Placebo in Patients With Depression \\ $\mathrm{N}=152$}

Design and baseline characteristics:

MC, R, DB, PC, flexible-dose-escalating study

Mean age: 56 years

Etiology of ERD: major depressive disorder (untreated)

Duration of ERD: 5.7 years

Severity of depression: mild $61 \%$, moderate $35 \%$, severe $4 \%$, mean HAM-D score 16.9

Drug regimen and duration:

Sildenafil $25 \mathrm{mg}-100 \mathrm{mg}(\mathrm{N}=74)$

Placebo $(\mathrm{N}=78)$

Duration: 12 weeks

Outcomes measures: global efficacy questions, IIEF erectile domain function, treatment response: yes to global efficacy questions $1-2$ and score $>21$ on erectile function domain of IIEF questionnaire, HAM-D: Beck Depression inventory, life satisfaction checklist

Results:

ERD treatment responders

Sildenafil vs. placebo

$73 \%$ vs. $14 \%$

Effect on depression measures

$\downarrow$ in HAM-D scores of 10.6 and 2.3 in treatment responders and nonresponders, respectively, regardless of treatment.

$76 \%$ of responders showed $\mathrm{a} \geq 50 \%$ decline in HAM-D scores vs. $14 \%$ of nonresponders

Life satisfaction improved in responders

Comments:

- Sildenafil was effective in this group of depressed men.

- Successful treatment was associated with improvement in depression scores and quality of life.

- Headache, dyspepsia, flushing, and abnormal vision were most frequent adverse events.

Nurnberg $^{52}$ (2003) Sildenafil vs. Placebo in Patients With Depression $\quad \mathrm{N}=90$

Design and baseline characteristics:

R, PC, DB, parallel group, flexible-dose study

Mean age: 45 years

Etiology of ERD: secondary to SSRI antidepressant treatment

Subjects in remission from depression

Mean SSRI use: 27 months

Drug regimen and duration:

Sildenafil $25 \mathrm{mg}-100 \mathrm{mg}$

Placebo

Duration: 6 weeks

Average 5 doses per treatment group

Outcomes measures: CGI-SF; IIEF erectile function; Arizona Sexual

Experience Scale; HAM-D

Results:

Sildenafil vs. placebo

Improvement in CGI-SF (primary measure)

$54.5 \%$ vs. $4.4 \%, P<0.001$

IIEF erectile function and other overall satisfaction measures were significantly improved for sildenafil subjects vs. placebo.

Comments:

- Mean depression scores remained constant and were consistent with remission.

- Headache, dyspepsia, flushing, nasal congestion, palpitations, insomnia, and abnormal vision were most frequent adverse events.

CGI-SF = Clinical Global Impression-Sexual Function; $D B=$ double blind; ERD=erectile dysfunction; HAM-D =Hamilton Depression Scale; HTN=hypertension;

$I I E F=$ International Index of Erectile Function; $M C=$ multicenter; $P C=$ placebo controlled; $Q=$ question; $R=$ randomized; $S C I=$ spinal cord injury;

SEP =Sexual Encounter Profile; SHIM=Sexual Health Inventory for Men; SSRI=selective serotonin reuptake inhibitor.

IIEF Question 3 or SEP Question 2: "When you attempted intercourse how often were you able to penetrate your partner?" IIEF Question 4 or SEP Question 3: "During sexual intercourse, how often were you able to maintain your erection after you had penetrated your partner?"; global efficacy question 1: "Did treatment improve your erections"; global efficacy question 2: "Did treatment improve your ability to have sexual intercourse?" 


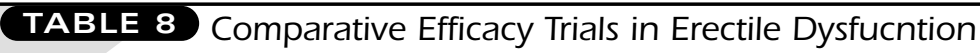

\begin{tabular}{|c|c|}
\hline $\begin{array}{r}\text { Shokeir }^{53}(2000) \\
\text { Intracavernous Injection PGEl vs. MUSE (Trar } \\
\text { Penile Insert } N=60\end{array}$ & \multirow{3}{*}{$\begin{array}{l}\text { maximum 1,000 mcg (transurethral alprostadil group was also offered option } \\
\text { of ACTIS penile ring); intracavernous alprostadil maximum } 40 \text { mcg } \\
\text { At-home, 3-week treatment phase at optimal transurethral alprostadil or intra- } \\
\text { cavernous alprostadil dose ( } \mathrm{N}=68 \text { ); patients were then crossed over to repeat } \\
\text { in office and at home phase with the alternate treatment. } \\
\text { Outcomes measure: IIEF erectile domain scores, physician and patient } \\
\text { assessments of erection quality, at least } 1 \text { erection sufficient for intercourse, } \\
\text { at least } 75 \% \text { successful ( } 75 \% \text { of all attempts are successful) }\end{array}$} \\
\hline RD of va & \\
\hline \multirow{2}{*}{$\begin{array}{l}\text { Drug regimen and duration: } \\
\text { ICI PGEl } 20 \mathrm{mcg}(\mathrm{N}=30) \\
\text { Transurethral alprostadil } 500 \mathrm{mcg}-1,000 \mathrm{mcg}(\mathrm{N}=30) \\
\text { Duration: } 3 \text { weeks }\end{array}$} & \\
\hline & \multirow{5}{*}{$\begin{array}{l}\text { Results: } \\
\text { In-office titration } \\
\text { - \% patients with erection grade } 3 \\
\text { - Intracavernous alprostadil had significantly more patients with erection } \\
\text { sufficient for intercourse than transurethral alprostadil ( } 62 \% \mathrm{vs.} 20 \% \text {, } \\
\text { P<0.0001). } \\
\text { - Average dose transurethral alprostadil } 921 \mathrm{mcg} \text {, average dose intracav- } \\
\text { ernous alprostadil } 27 \mathrm{mcg} \\
\text { At-home phase } \\
\text { - Intracavernous alprostadil had significantly more patients with at least } \\
1 \text { erection sufficient for intercourse than transurethral alprostadil (92\% vs. } \\
62 \% ; P<0.0001 \text { ). } \\
\text { - More patients on intracavernous alprostadil were at least } 75 \% \text { successful on } \\
\text { all attempts than those taking transurethral alprostadil ( } 75 \% \text { vs. } 37 \% \text {; } \\
P<0.0001 \text { ). } \\
\text { - IIEF scores were significantly higher with intracavernous alprostadil than } \\
\text { with transurethral alprostadil ( } P<0.0001 \text { ). }\end{array}$} \\
\hline s: erectile assessment s & \\
\hline $\begin{array}{l}\text { Results: } \\
\text { - Intercourse occurred after } 85 \% \text { of all ICI PGEl administrations vs. } 55 \% \text { of } \\
\text { all transurethral alprostadil administrations. } \\
\text { - At least } 1 \text { successful intercourse for } 87 \% \text { of ICI PGEl subjects vs. } 53 \% \text { of } \\
\text { transurethral alprostadil subjects }(P<0.05)\end{array}$ & \\
\hline $\begin{array}{l}\text { Comments: } \\
\text { - } 67 \% \text { discontinuation rate with ICI PGEl approximately 50\% due to penile } \\
\text { pain } \\
\text { - } 17 \% \text { discontinuation with transurethral alprostadil with lower rate of penile } \\
\text { pain } \\
\text { - No reports of priapism }\end{array}$ & \\
\hline & \\
\hline $\begin{array}{l}\text { MUSE (Transurethral Alprostadil) vs. Edex (In } \\
\qquad \mathrm{N}=111\end{array}$ & \multirow{4}{*}{$\begin{array}{l}\text { Comments: } \\
\text { - Penile pain common to both intracavernous alprostadil and transurethral } \\
\text { alprostadil (20\%-34\%) } \\
\text { - Application site reaction more common with transurethral alprostadil } 10 \% \text { - } \\
15 \% \text { than with intracavernous alprostadil ( } 2 \%-4 \%) \\
\text { - Intracavernous alprostadil had a } 2 \%-3 \% \text { rate of prolonged erections as } \\
\text { compared with none in the transurethral alprostadil group. }\end{array}$} \\
\hline igl & \\
\hline & \\
\hline $\begin{array}{l}\text { ion phase ( } 1-14 \text { days) to } \\
\text { urethral alprostadil dose }\end{array}$ & \\
\hline
\end{tabular}

in IIEF score from baseline, normalization of IIEF erectile function domain, mean improvement in erectile function score, and percentage improvement over baseline, to name a few.

\section{Sexual Health Inventory for Men (SHIM)}

The SHIM is an abbreviated version of the IIEF questionnaire and was designed to allow a more rapid diagnosis of ERD and assignment of ERD severity. The instrument has 6 questions, with a maximum score of 30. ERD is present if the SHIM score is 21 or less. The SHIM primarily measures erectile function, and it does not address measures of orgasmic function, libido, and satisfaction.

\section{Sexual Encounter Profile (SEP) Diary}

Assessments of individual sexual encounters are provided by SEP diaries. The SEP diary is intended to be an immediate-recall diary of encounters. The diaries contain 6 questions for the patient and 4 questions for the partner. SEP questions 2 and 3 are common outcomes measures in efficacy studies, and they are very similar to questions 3 and 4 of the IIEF erectile dysfunction domain. However, the SEP questions are answered yes or no while the IIEF questions are assigned a numerical score.

\section{Global Assessment or Global Efficacy Questions}

Global assessment or efficacy questions are often used as secondary outcomes measures. The 2 most common questions are: "Did this treatment improve your erections?" and "Did treatment improve your ability to have sexual intercourse?"

\section{Clinical Efficacy Summary General ERD Population: PDE5 Inhibitors}

Sildenafil, vardenafil, and tadalafil significantly improve IIEF erectile function domain scores and improve erection quality as compared with placebo in large, double-blind, randomized, controlled trials in the general ERD population. ${ }^{31-35}$ There are several outcomes measurements reported in ERD clinical studies, 


\section{TABLE $9 \longdiv { \text { Miscellaneous Studies: Failed Previous Erectile Dysfunction Therapy } }$}

\section{Engel $^{55} 1998$}

MUSE (Transurethral Alprostadil) in ICI PGE1, Papaverine or Phentolamine Failures $\mathrm{N}=452$

Design and baseline characteristics:

PC, DB, retrospective study

Included some ERD patients not responsive to ICI of alprostadil (PGE1), 95/452; papaverine or phentolamine

Mean age: 60 years

\section{Dosage regimen and duration:}

In-office phase

Titration to response with $125 \mathrm{mcg}-1,000 \mathrm{mcg}$ of transurethral alprostadil At-home phase

3 months treatment with transurethral alprostadil or placebo

Outcomes measures: Physician and patient assessment of erection, patient diaries

\section{Results:}

$58 \%$ of patients previously unresponsive to ICI PGEl achieved an adequate erection at least once during the in-office phase

$47 \%$ of this group reported at least 1 successful intercourse during the athome phase vs. $12 \%$ for placebo.

Most efficacy measures were significantly higher for transurethral alprostadil than placebo.

\section{Comments:}

- Number of placebo administrations was much lower than the number of transurethral alprostadil administrations

- Penile pain was the most common adverse event (7.8\%)

Shabsigh $^{56}(2000)$
Edex (Intracavernous Alprostadil) in Sildenafil Failures
$\mathrm{N}=134$

Design and baseline characteristics:

MC, open-label study

Mean age: 59 years

Etiology of ERD: organic 92\%-98\%

\section{Dosage regimen and duration:}

Subjects treated with sildenafil $5 \mathrm{mg}-100 \mathrm{mg}$ for 4 weeks $(\mathrm{N}=134)$

Nonresponders or partial responders $(\mathrm{N}=67)$ with IIEF score of 3 or less given intracavernous alprostadil and titrated in-office until response (up to $40 \mathrm{mcg}$ ) At-home phase

6 weeks of treatment with on demand intracavernous alprostadil
Outcomes measures: IIEF Q.3 (penetration) and Q.4 (maintenance of erection for successful intercourse), erectile response score (physician and patient assessment)

Results:

In-office phase

Mean dose of intracavernous alprostadil $28 \mathrm{mcg}$

$94 \%$ of patients were able to achieve an adequate erectile response as per physician assessment.

At-home phase

$88 \%$ of intracavernous alprostadil subjects in 6-week at-home phase had erections adequate for intercourse.

$89 \%$ and $85 \%$ of patients had an improvement of 1 or more in IIEF score for Q.3 and Q.4, respectively.

\section{Comments:}

- Most frequent adverse events with intracavernous alprostadil were pain, paresthesias, and influenza-like symptoms.

- Subjects were considered to be sildenafil "failures" even if they had adequate response for $50 \%$ of all attempts.

Carson $^{57}$ (2003) Vardenafil in Sildenafil Failures $\quad \mathrm{N}=463$

Design and baseline characteristics:

DB, MC, PC, flexible-dose study

ERD severity: moderate to severe

Sildenafil failures defined as failure with sildenafil on at least 4 of 6 attempts with at least 1 failure at the $100 \mathrm{mg}$ dosage level

\section{Drug regimen and duration:}

Vardenafil $10 \mathrm{mg}(\mathrm{N}=231)$; titration to $5 \mathrm{mg}$ or $20 \mathrm{mg}$ could occur at 4 week intervals

Placebo $(\mathrm{N}=226)$

Duration: 12 weeks

Outcomes measures: IIEF erectile domain scores, SEP Q.2 (penetration), SEP Q.3 (maintenance of erection for successful intercourse), global assessment Q.1 (improvement of erections)
Results:
Vardenafil vs. placebo
IIEF scores
$46 \%$ vs. $16 \%, P<0.001$
17.6 vs. $10.5, P<0.001$
SEP Q.2

$$
62.3 \% \text { vs. } 29.9 \%, P<0.001
$$
Improvement in erections $61.6 \%$ vs. $14.7 \%, P<0.001$

\section{Comments:}

- Most common adverse events were headache, dyspepsia, nasal congestion, and flushing.

$D B=$ double blind; $E R D=$ erectile dysfunction; $I C I=$ intracavernous injection; $I I E F=$ International Index of Erectile Function; $M C=$ multicenter; $M U S E=M e d i c a t e d ~ U r e t h r a l$ System for Erection; PC=placebo controlled; PGE1= prostaglandin E1; Q=question; SEP=Sexual Encounter Profile.

IIEF Question 3 or SEP Question 2: "When you attempted intercourse how often were you able to penetrate your partner?" IIEF Question 4 or SEP Question 3 : "During sexual intercourse, how often were you able to maintain your erection after you had penetrated your partner?"; Global efficacy question 1: "Did treatment improve your erections?"

but perhaps the most meaningful improvement to the patient is the rate of successful intercourse. In a meta-analysis of 14 randomized, placebo-controlled, flexible-dose studies, the subjects on sildenafil $25 \mathrm{mg}$ to $100 \mathrm{mg}$ had a successful intercourse rate of $57 \%$ as compared with a rate of $21 \%$ with placebo. ${ }^{31}$ Combined data from 2 fixed-dose sildenafil studies showed a successful intercourse rate of $43 \%, 50 \%$, and $51 \%$ for the $25 \mathrm{mg}, 50 \mathrm{mg}$, and $100 \mathrm{mg}$ dose, respectively, as compared with the placebo group, which had rates of $14 \%$ to $17 \% .^{31}$ In general, higher sildenafil doses were associated with higher efficacy rates. Also included in the meta-analysis were additional analyses examining efficacy in subgroups stratified by age, race, ERD baseline severity, and ERD etiology. Sildenafil was as efficacious in the Asian and African American subjects as in 
whites, who comprise the majority of subjects in ERD studies. While the rate of successful intercourse varied depending on age, ERD severity, and ERD etiology, sildenafil use resulted in significantly greater rates for each subgroup as compared with placebo. ${ }^{31}$

In one large, randomized, fixed-dose study, vardenafil, at doses ranging from $5 \mathrm{mg}$ to $20 \mathrm{mg}$, was able to produce a significantly greater rate of erections adequate for intercourse$50 \%$ to $65 \%$ - compared with a placebo rate of $32 \%$, which is higher than the reported placebo average of $20 \%$. The high placebo rate seen in this study is intriguing because 30\% to $45 \%$ of subjects were classified by investigators as having severe ERD. An increase in efficacy was seen with increasing vardenafil dose. ${ }^{19}$ Vardenafil significantly improved IIEF erectile function domain scores as compared with placebo regardless of patient age, ERD etiology, or baseline ERD severity. ${ }^{33}$

In an integrated analysis of 5 multicenter, double-blind, randomized, fixed-dose studies, tadalafil $2.5 \mathrm{mg}, 5 \mathrm{mg}, 10 \mathrm{mg}$, and $20 \mathrm{mg}$ resulted in significantly higher rates of successful intercourse, $36 \%, 42 \%, 61 \%$, and $75 \%$, respectively, compared with a placebo rate of $32 \% .{ }^{34}$ Another multicenter, doubleblind, randomized, fixed-dose study compared the duration of efficacy of $20 \mathrm{mg}$ tadalafil with placebo..$^{35}$ At 24 hours postadministration, a 53\% rate of successful intercourse attempts was reported in the tadalafil group compared with a $29 \%$ rate in the placebo group. Tadalafil remained significantly more efficacious than placebo at 36 hours postdose, with a rate of $59 \%$ compared with $28 \%{ }^{35}$ The results of this study confirm the long duration of tadalafil, which would be anticipated from its prolonged half-life of 17 hours.

No comparative studies have been done to assess relative efficacy of any one PDE5 inhibitor to another. Until large comparative studies prove otherwise, the efficacy of these products seems roughly equivalent; however, direct comparisons of efficacy and safety should not be made, given the many variables present in populations studied and outcomes measures used.

\section{General ERD Population: Alprostadil}

Intracavernous and transurethral administration of alprostadil, while not usually considered first-line therapy, is also effective in the management of ERD in the general population. In alprostadil studies, efficacy is most often measured by physician and patient assessment of erection quality. In one large, multicenter, randomized, fixed-dose study, intracavernous administration of alprostadil at doses of $2.5 \mathrm{mcg}, 5 \mathrm{mcg}$, $10 \mathrm{mcg}$, and $20 \mathrm{mcg}$ resulted in $20 \%, 30 \%, 35 \%$, and $50 \%$, respectively, of men achieving full erections. ${ }^{36}$ The mean duration of erection was 37 minutes, and the duration was related to dose. Five men had prolonged erections; in 2 men, the erections lasted 4 hours or more. Penile pain was reported by $23 \%$ of intracavernous alprostadil subjects. In a 6-month self-injection extension of the study, the intracavernous alprostadil responders reported being able to have intercourse after the injections $94 \%$ of the time..$^{36}$ In another placebocontrolled crossover study, intracavernous alprostadil $1 \mathrm{mcg}$ to $40 \mathrm{mcg}$ resulted in $73 \%$ to $74 \%$ of erections deemed adequate for intercourse (patient assessment) as compared with rates of $7 \%$ to $13 \%$ for placebo. ${ }^{37}$ The median duration of erection was 59 minutes, and prolonged erections lasting 4 to 6 hours were noted in $3 \%$ of subjects taking intracavernous alprostadil. The average intracavernous alprostadil dose was not reported in this study. Penile pain and bleeding were other common adverse events. ${ }^{37}$

Transurethral alprostadil was significantly more effective than placebo in 2 double-blind, placebo-controlled studies. ${ }^{38,39}$ In these studies, using transuretharal alprostadil doses ranging from $125 \mathrm{mcg}$ to $1,000 \mathrm{mcg}$, the rates of erections deemed adequate for intercourse were $49 \%$ to $66 \%$. In the at-home phase of one of the studies, transuretharal alprostadil resulted in a successful intercourse rate of $65 \%$ compared with a placebo rate of $19 \%$. Incidence of penile pain ranged from 9\% to 19\%, hypotension was 3\%, and there were no reports of priapism or prolonged erections. ${ }^{38,39}$

Two open-label studies compared the efficacy of transurethral alprostadil versus intracavernous alprostadil. In one study, the intracavernous alprostadil product was an extemporaneous preparation ${ }^{53}$; in the other, the Edex preparation was used. ${ }^{54}$ In both studies, intracavernous injections of alprostadil resulted in significantly higher erectile assessment scores or IIEF erectile function domain scores as compared with transurethral alprostadil. In one study, transurethral alprostadil was better tolerated with a lower discontinuation rate due to penile pain ${ }^{53}$; however, the other study reported similar rates of penile pain and a marked patient preference for injection over transurethral therapy. ${ }^{54}$

\section{General ERD Population: Failures on Previous ERD Therapy}

One open-label, multicenter study reported that intracavernous alprostadil, in doses up to $40 \mathrm{mcg}$, was effective in failures with sildenafil therapy. In this study, sildenafil failures had a score of 1.2 or less on the IIEF erectile domain questions 3 and 4 . A score of 1 means that sildenafil was almost never or never effective. Use of intracavernous alprostadil resulted in the IIEF scores improving by 2.75 to 2.63 points for $85 \%$ to $90 \%$ of patients. Penile pain was present in $30 \%$ of all intracavernous alprostadil subjects. ${ }^{56}$

One open-label, multicenter study examined the efficacy of vardenafil $5 \mathrm{mg}$ to $20 \mathrm{mg}$ in the treatment of ERD in 134 patients determined to be unresponsive to sildenafil. Unresponsiveness was defined as failure with sildenafil on at least 4 out of 6 attempts, with at least one of those attempts at the $100 \mathrm{mg}$ dosage level. Sildenafil failures were randomized to receive either vardenafil $(\mathrm{N}=231)$ or placebo $(\mathrm{N}=226)$ for a treatment period of 12 weeks. Vardenafil use resulted in significantly higher IIEF erectile domain scores than placebo 
and higher rates of maintenance of erection sufficient for intercourse $(46 \%$ vardenafil versus $16 \%$ placebo; $P<.001)$. Overall, $62 \%$ of vardenafil subjects stated that their erections were improved compared with $15 \%$ of those in the placebo group. ${ }^{57}$

\section{Special Populations \\ Diabetes}

Several double-blind, placebo-controlled studies have been performed to evaluate the efficacy of sildenafil, vardenafil, and tadalafil in the management of ERD associated with type 1 and type 2 diabetes. ${ }^{40-44}$ No direct comparative studies have been performed to assess relative efficacy of one PDE5 inhibitor to another. However, well-designed studies have reported the following rates of successful intercourse: sildenafil $48 \%$ versus placebo $12 \%$; vardenafil $49 \%$ to $54 \%$ versus $23 \%$; tadalafil $28 \%$ to $29 \%$ versus $1.9 \% .^{44}$ The lower success rate seen with tadalafil may be due to the high percentage $(72 \%)$ of patients with severe ERD enrolled in the study.

\section{Postprostatectomy}

As with diabetes, several clinical studies have assessed the efficacy of all of the currently available PDE5 inhibitors in the management of ERD postprostatectomy. In this patient population, response to treatment is dependent on subject age, baseline ERD severity, and the type of prostatectomy surgery. In general, bilateral nerve-sparing surgery is associated with the best chance for response with non-nerve-sparing procedures having the lowest response to therapy. However all PDE5 inhibitors are potentially effective in the management of postprostatectomy ERD. ${ }^{45-49}$

\section{Post-Spinal-Cord Injury}

Of the PDE5 inhibitors, only sildenafil has been studied in the management of ERD resulting from spinal cord injury. This patient population differs not only in the etiology of ERD but also in age since the average spinal cord injury patient in clinical studies is much younger (38 years) as compared with the ERD patient in the general population (56 years). In one randomized, placebo-controlled crossover study in 178 spinal cord injury patients, doses of sildenafil $50 \mathrm{mg}$ to $100 \mathrm{mg}$ resulted in an intercourse success rate of $55 \%$ versus $0 \%$ for placebo. Thus, success rates for sildenafil in ERD secondary to spinal cord injury approach rates seen in subjects with other comorbid conditions..$^{50}$

\section{Depression}

One double-blind, placebo-controlled study has evaluated the efficacy of sildenafil in the management of ERD in patients with depression. Most patients in this study had a diagnosis of mild or moderate major depression and were not treated with antidepressants. Sildenafil $25 \mathrm{mg}$ to $100 \mathrm{mg}$ or placebo was given for 12 weeks. At the end of the study, significantly more patients on sildenafil than placebo ( $73 \%$ versus $14 \%$ ) had a treatment response as defined by IIEF erectile function treatment scores and positive responses to 2 global efficacy questions. Successful treatment was also associated with an improvement in Hamilton Depression scores and quality-of-life measures. ${ }^{51}$

Sildenafil was more effective than placebo ( $55 \%$ versus $4.4 \% ; P<.001)$ in improving Clinical Global Impression-Sexual Function scores in a study with 90 patients with ERD secondary to treatment with selective serotonin reuptake inhibitor antidepressants. All patients were in remission from major depression and remained on antidepressants during treatment with sildenafil for 6 weeks. ${ }^{52}$

\section{Effectiveness Studies}

Overall, in controlled clinical studies, sildenafil has an efficacy rate of roughly $60 \%$ in the broad ERD population. ${ }^{31}$ However, in the real-world setting, refill rates for sildenafil are not as high as would be expected. Of patients tracked for 1 year, only $52 \%$ filled a second prescription during that 12 -month period and $31 \%$ filled greater than 7 prescriptions..$^{58}$ In another study, patients in a clinic were followed for 2 years to evaluate their response to sildenafil..$^{59}$ Two surveys were conducted. The first survey went to 200 men who had recently been given a prescription for sildenafil. Of these 200 men, only 151 (75\%) actually tried the drug. Of those who tried the drug, an overall success rate of $74 \%$ was reported. The most common doses used were $50 \mathrm{mg}(\mathrm{n}=88)$ and $100 \mathrm{mg}(\mathrm{n}=61)$. While $38 \%$ of patients reported side effects, none discontinued therapy from drug intolerance. Two years later, a second survey was sent out; only 82 patients participated. Of those patients, $17 \%$ discontinued because of loss of efficacy and 20\% needed to increase their dose by $50 \mathrm{mg}$. There was no correlation between frequency of use and the need to increase the dose. While the authors concluded that tachyphylaxis to sildenafil was responsible for study results, it is not clear if this is the case. ${ }^{59}$ Other reasons for reduced effect over time could have included psychological factors as well as worsening of underlying comorbid conditions, especially progressive vascular disease or poorly controlled diabetes.

Efficacy results in controlled clinical studies are rarely, if ever, duplicated in the real-world setting, and the experience with ERD is no different. However, McCullough et al. did report on several studies designed to identify and improve success rates with sildenafil therapy ${ }^{60}$ The intensive disease management approach utilized in one of the studies yielded impressive results. Overall, 55\% of men not previously successful with sildenafil became successful after intensive reeducation and counseling, which included regular follow-up visits with information as to how to take the drug, titration to maximum dose, and a minimum trial of 8 attempts for efficacy assessment. Controlling risk factors for ERD as recommended in current treatment guidelines also was a successful strategy, although 
Clinical Monograph for Drug Formulary Review: Erectile Dysfunction Agents

TABLE 10 PDE5 Inhibitors: Selected Adverse Events Occurring >2\% in Placebo-Controlled Studies ${ }^{14-16}$

\begin{tabular}{l|c|c|c}
\hline Adverse Event & Sildenafil/Placebo (\%) & Vardenafil/Placebo (\%) & Tadalafil/Placebo (\%) \\
\hline Headache & $16 / 4$ & $15 / 4$ & $11-15 / 5$ \\
\hline Flushing & $10 / 1$ & $11 / 1$ & $4-10 / 1$ \\
\hline Rhinitis/nasal congestion & $4 / 2$ & $9 / 3$ & $4-10 / 1$ \\
\hline Dyspepsia & $7 / 2$ & $<2$ & Rare, 1 episode reported \\
\hline Abnormal vision & $3 / 0$ & $3 / 1$ & NR \\
\hline Sinusitis & NR & $2 / 1$ & NR \\
\hline Increased creatinine kinase & NR & $3 / 2$ & NR \\
\hline Flu syndrome & NR & $2 / 1$ & NR \\
\hline Dizziness & $2 / 1$ & $<2$ & $3-6 / 3$ \\
\hline Back pain & $<2$ & $<2$ & $1-4 / 1$ \\
\hline Myalgia & $<2$ & & \\
\hline NR=not reported & & & \\
\hline
\end{tabular}

men with only 1 risk factor were more likely to respond to intervention than men with multiple risk factors. ${ }^{60}$

\section{Adverse Events PDE5 Inhibitors}

Tadalafil, sildenafil, and vardenafil were well tolerated in clinical studies with headache, flushing, and dyspepsia occurring as the most common adverse events. There are no comparative safety data to compare rates of common adverse events, but based on the rates seen in placebo-controlled studies, there appears to be little difference in safety profiles for these most commonly reported events. Discontinuations secondary to adverse events were low for all 3 PDE5 inhibitors, ranging from $1 \%$ to $5 \%$. Changes in color vision, which has been reported with sildenafil use, are less frequent with vardenafil and rarely reported with tadalafil. However, tadalafil does seem to be associated with more reports of myalgia and back pain than vardenafil or sildenafil. The muscle aches and back pain usually occur within 12 to 24 hours after tadalafil administration and resolve within 48 hours. Approximately $0.5 \%$ of patients discontinued tadalafil because of back pain or myalgia. ${ }^{14-16}$

\section{Serious Cardiac Events}

Cardiac mortality rates in the tadalafil clinical study database ( $N>4,000$ subjects) are consistent with the expected rate in a male population. Across all studies, the incidence rate of myocardial infarction was 0.43 per 100 patient years in the tadalafil-treated patients compared with 0.6 per 100 patient years in the placebo-treated population, which was also consistent with the incidence rate observed with an age-standardized male population. ${ }^{61}$

The cardiac safety of sildenafil has been extensively studied.

\section{TABLE 11 Selected Adverse Events: Intracavernous} and Transurethral Alprostadil ${ }^{11-13}$

\begin{tabular}{|c|c|c|c|}
\hline Adverse Event & $\begin{array}{c}\text { Caverject } \\
\text { (Intracavernous } \\
\text { Alprostadil) } \\
(\%)\end{array}$ & \begin{tabular}{|c|} 
Edex \\
(Intracavernous \\
Alprostadil) \\
$(\%)$
\end{tabular} & $\begin{array}{c}\text { MUSE } \\
\text { (Transurethral } \\
\text { Alprostadil) } \\
(\%)\end{array}$ \\
\hline \multicolumn{4}{|l|}{ Local side effects } \\
\hline Injection site ecchymosis & 2 & 4 & NR \\
\hline Injection site hematoma & 3 & 5 & NR \\
\hline Penile edema & 1 & 2 & NR \\
\hline Penile fibrosis & 3 & 5 & NR \\
\hline Penile pain & 37 & 35 & 32 \\
\hline Penile rash & 1 & NR & NR \\
\hline Penis disorder & 3 & 3 & NR \\
\hline Prolonged erection & 4 & $4 *$ & 0.3 \\
\hline Priapism & 0.4 & $<1 \dagger$ & $<0.1$ \\
\hline Testicular pain & NR & NR & 5 \\
\hline Urethral bleeding_minor & NR & NR & 5 \\
\hline Urethral burning & NR & NR & 12 \\
\hline \multicolumn{4}{|l|}{ Systemic side effects } \\
\hline Headache & 2 & 2 & 3 \\
\hline Dizziness & 1 & NR & 2 \\
\hline Hypotension & $<1$ & $<1$ & 3 \\
\hline Back pain & 1 & 2 & 2 \\
\hline Upper respiratory infection & 4 & 2 & 3 \\
\hline Flu syndrome & 2 & NR & 4 \\
\hline
\end{tabular}




\begin{tabular}{|c|c|c|c|}
\hline & Sildenafil & Vardenafil & Tadalafil \\
\hline \multicolumn{4}{|l|}{ Contraindications } \\
\hline Nitrates & $\mathrm{X}$ & $\mathrm{X}$ & $\mathrm{X}$ \\
\hline Alpha-blockers & $*$ & $\mathrm{X}$ & $\mathrm{X}^{\dagger}$ \\
\hline Hypersensitivity & $\mathrm{X}$ & $\mathrm{X}$ & $\mathrm{X}$ \\
\hline \multicolumn{4}{|l|}{ Warnings and precautions } \\
\hline Cardiovascular effects & $\mathrm{X}$ & $\mathrm{X}$ & $\mathrm{X}$ \\
\hline $\begin{array}{l}\text { Left ventricular outflow } \\
\text { obstruction }\end{array}$ & $\mathrm{X}$ & $\mathrm{X}$ & $\mathrm{X}$ \\
\hline Blood pressure effects & $\mathrm{X}$ & $\mathrm{X}$ & $\mathrm{X}$ \\
\hline Strong CYP3A4 inhibitors & $\mathrm{X}$ & $\mathrm{X}$ & $\mathrm{X}$ \\
\hline Priapism & $\mathrm{X}$ & $\mathrm{X}$ & $\mathrm{X}$ \\
\hline Concurrent alpha blocker & $\mathrm{X}$ & \begin{tabular}{|c|} 
See \\
contraindications
\end{tabular} & $\begin{array}{c}\text { See } \\
\text { contraindications }\end{array}$ \\
\hline Hepatic impairment & & $\mathrm{X}$ & $\mathrm{X}$ \\
\hline QT prolongation & & $\mathrm{X}$ & \\
\hline Renal impairment & & $\mathrm{X}$ & $\mathrm{X}$ \\
\hline $\begin{array}{l}\text { Bleeding disorders or active } \\
\text { peptic ulceration }\end{array}$ & $\mathrm{X}$ & $\mathrm{X}$ & $\mathrm{X}$ \\
\hline $\begin{array}{l}\text { Anatomical deformities of } \\
\text { the penis }\end{array}$ & $\mathrm{X}$ & $\mathrm{X}$ & $\mathrm{x}$ \\
\hline $\begin{array}{l}\text { Conditions that predispose } \\
\text { to priapism (e.g., sickle cell } \\
\text { anemia, multiple myeloma, } \\
\text { leukemia) }\end{array}$ & $\mathrm{X}$ & $\mathrm{X}$ & $\mathrm{X}$ \\
\hline $\begin{array}{l}\text { Combination with other } \\
\text { therapies for erectile } \\
\text { dysfunction }\end{array}$ & $\mathrm{X}$ & & \\
\hline \multicolumn{4}{|c|}{$\begin{array}{l}\text { * Sildenafil doses }>25 \mathrm{mg} \text { should not be given within } 4 \text { hours of administration of an } \\
\text { alpha-blocker. } \\
\text { † Except for tamsulosin } 0.4 \mathrm{mg} \text { once daily. }\end{array}$} \\
\hline
\end{tabular}

Pooled results from 53 clinical studies indicated no difference between the incidence of death or myocardial infarction in men with ERD receiving sildenafil or placebo. ${ }^{62}$ In a United Kingdom study, 5,601 patients with ERD showed no evidence of increased risk of myocardial or ischemic heart disease during the first 4.9 months of sildenafil therapy. ${ }^{63}$ This low risk is supported by open-label safety data from subjects who have been taking sildenafil for up to 4.5 years. ${ }^{64}$

Vardenafil has been shown to prolong the cardiac conduction as evidenced by a prolonged QT interval at therapeutic and supratherapeutic doses (Section VII, Contraindications/ Precautions). ${ }^{15}$

\section{Intracavernosal and Transurethral Alprostadil}

The type and degree of side effects reported in the 2 intracavernous alprostadil formulations are very similar. ${ }^{11-13}$ No
TABLE 13 Contraindications, Warnings, and Precautions for Caverject (Intracavernous Alprostadil), Edex (Intracavernous Alprostadil), and MUSE (Transurethral Alprostadil) ${ }^{1-13}$

\begin{tabular}{l|c|c|c}
\hline & $\begin{array}{c}\text { Intracavernous } \\
\text { Alprostadil }\end{array}$ & $\begin{array}{c}\text { Intracavernous } \\
\text { Alprostadil }\end{array}$ & $\begin{array}{c}\text { Transurethral } \\
\text { Alprostadil }\end{array}$ \\
\hline Contraindication & $\mathrm{X}$ & $\mathrm{X}$ & $\mathrm{X}$ \\
\hline $\begin{array}{l}\text { Known hypersensitivity } \\
\text { to alprostadil }\end{array}$ & $\mathrm{X}$ & $\mathrm{X}$ & $\mathrm{X}$ \\
\hline $\begin{array}{l}\text { Conditions that may } \\
\text { predispose the patient } \\
\text { to priapism }\end{array}$ & $\mathrm{X}$ & $\mathrm{X}$ & $\mathrm{X}$ \\
\hline $\begin{array}{l}\text { Anatomical deformation } \\
\text { of the penis }\end{array}$ & $\mathrm{X}$ & $\mathrm{X}$ & $\mathrm{X}$ \\
\hline $\begin{array}{l}\text { Males in whom sexual } \\
\text { activity is contraindicated }\end{array}$ & $\mathrm{X}$ & $\mathrm{X}$ & $\mathrm{X}$ \\
\hline $\begin{array}{c}\text { Sexual intercourse with a } \\
\text { pregnant woman unless a } \\
\text { condom barrier is used }\end{array}$ & $\mathrm{X}$ & $\mathrm{X}$ & $\mathrm{X}$ \\
\hline Penile implants & $\mathrm{X}$ & $\mathrm{X}$ & $\mathrm{X}$ \\
\hline Precautions & $\mathrm{X}$ & $\mathrm{X}$ & $\mathrm{X}$ \\
\hline Priapism & & & \\
\hline fibrosis & & & $\mathrm{X}$ \\
\hline Anticoagulant therapy & $\begin{array}{l}\text { Combination with other } \\
\text { vasoactive agents }\end{array}$ & & \\
\hline
\end{tabular}

controlled comparative studies are available that directly compared the adverse event rates of these 2 products. As might be expected from a penile injection, local side effects (ecchymosis, hematoma, edema, pain) are prominent with both. Transurethral alprostadil is also associated with a significant occurrence of penile pain, urethral burning, and bleeding. The 2 comparison studies that compared transurethral alprostadil with intracavernous alprostadil injections had conflicting results regarding penile pain and discontinuations due to adverse events. ${ }^{53,54}$ Prolonged erection or, in some cases, priapism, can occur with intracavernous alprostadil and transurethral alprostadil. ${ }^{11-13}$

Tables 10 and 11 display selected common adverse events as reported in respective product labeling for the currently marketed PDE5 inhibitors ${ }^{14-16}$ and the alprostadil intracavernosal and transurethral products. ${ }^{11-13}$ Direct comparisons between adverse events rates cannot be made as the event rates displayed are not derived from comparative studies.

\section{Contraindications/Precautions}

The contraindications, warnings, and precautions for sildenafil, tadalafil, and vardenafil are extremely similar (Table 12). Of note, vardenafil in therapeutic (10 $\mathrm{mg}$ ) and supratherapeutic 
TABLE 14 Drug and Food Interactions With PDE5 Inhibitors ${ }^{14-16}$

\begin{tabular}{|c|c|c|c|}
\hline & Sildenafil & Vardenafil & Tadalafil \\
\hline Administration with food & $\begin{array}{l}\text { Can be administered with food, but } \\
\text { high-fat meal slows absorption and } \\
\text { peak plasma concentrations }\end{array}$ & $\begin{array}{l}\text { Can be administered with food, but } \\
\text { high-fat meal slows absorption and } \\
\text { peak plasma concentrations }\end{array}$ & Can be administered with food \\
\hline Nitrates & Contraindicated & Contraindicated & Contraindicated \\
\hline Alpha-blockers & $\begin{array}{l}\text { Not contraindicated, but do not use } \\
\text { doses higher than } 25 \mathrm{mg} \text { within } \\
4 \text { hours of taking an alpha-blocker }\end{array}$ & Contraindicated & $\begin{array}{l}\text { Contraindicated except for tamsulosin } \\
\text { (Flomax) at the } 0.4 \mathrm{mg} \text { dose }\end{array}$ \\
\hline Class IA and III antiarrhythmics & No precautions & $\begin{array}{l}\text { Avoid concomitant use; vardenafil } \\
\text { shown to increase QT interval }\end{array}$ & No precautions \\
\hline $\begin{array}{l}\text { Strong CYP3A4 inhibitors (grapefruit } \\
\text { juice, erythromycin, ketoconazole, } \\
\text { itraconazole) }\end{array}$ & $25 \mathrm{mg}$ sildenafil recommended & $\begin{array}{l}5 \mathrm{mg} \text { of vardenafil no more frequently } \\
\text { than every } 24 \text { hours; reduce dose to } \\
2.5 \mathrm{mg} \text { with } 400 \mathrm{mg} \text { of ketoconazole } \\
\text { or itraconazole }\end{array}$ & $\begin{array}{l}10 \mathrm{mg} \text { of tadalafil no more frequently } \\
\text { than every } 72 \text { hours }\end{array}$ \\
\hline HIV protease inhibitors & $\begin{array}{l}\text { Ritonavir: } 25 \mathrm{mg} \text { of sildenafil no more } \\
\text { frequently than every } 48 \text { hours; } \\
\text { Saquinavir: an initial sildenafil dose of } \\
25 \mathrm{mg} \text { is recommended }\end{array}$ & $\begin{array}{l}\text { Ritonavir: } 2.5 \mathrm{mg} \text { vardenafil no more } \\
\text { frequently than every } 72 \text { hours } \\
\text { Indinavir: } 2.5 \mathrm{mg} \text { no more frequently } \\
\text { than every } 24 \text { hours }\end{array}$ & $\begin{array}{l}\text { Ritonavir: } 10 \text { mg of tadalafil no more } \\
\text { frequently than every } 72 \text { hours; dosage } \\
\text { applies to all HIV protease inhibitors }\end{array}$ \\
\hline
\end{tabular}

CYP=cytochrome P450 isoenzyme; HIV = human immunodeficiency virus; PDE5= phosphodiesterase type 5 .

\section{TABLE 15 Indications and Dosage for PDE5 Inhibitors}

\begin{tabular}{|c|c|c|c|}
\hline & Sildenafil & Vardenafil & Tadalafil \\
\hline FDA indication & \multicolumn{3}{|c|}{ All PDE 5 inhibitors are indicated for the treatment of erectile dysfunction } \\
\hline Usual adult dose & $\begin{array}{l}50 \mathrm{mg} \text { approximately } 1 \text { hour before } \\
\text { sexual activity; the dose may be increased } \\
\text { to } 100 \mathrm{mg} \text { or decreased to } 25 \mathrm{mg} \text { based } \\
\text { on efficacy and side effects; the maximum } \\
\text { dosing frequency is once daily }\end{array}$ & $\begin{array}{l}10 \mathrm{mg} \text { approximately } 1 \text { hour before } \\
\text { sexual activity; the dose may be increased } \\
\text { to } 20 \mathrm{mg} \text { or decreased to } 5 \mathrm{mg} \text { based on } \\
\text { efficacy and side effects; the maximum } \\
\text { dosing frequency is once daily }\end{array}$ & $\begin{array}{l}10 \text { mg taken prior to sexual activity; } \\
\text { the dose may be increased to } 20 \mathrm{mg} \text { or } \\
\text { decreased to } 5 \mathrm{mg} \text { based on tolerability } \\
\text { and efficacy; maximum frequency is } \\
\text { once daily }\end{array}$ \\
\hline Hepatic impairment & $\begin{array}{l}\text { A starting dose of } 25 \mathrm{mg} \text { is } \\
\text { recommended }\end{array}$ & $\begin{array}{l}\text { Not recommended for patients with } \\
\text { severe hepatic impairment; } 5 \mathrm{mg} \text { dose } \\
\text { recommended for moderate hepatic } \\
\text { impairment, and the maximum dose } \\
\text { should not exceed } 10 \mathrm{mg} \text {; no dose } \\
\text { adjustment is necessary in patients } \\
\text { with mild hepatic impairment }\end{array}$ & $\begin{array}{l}\text { Not recommended for patients with } \\
\text { severe hepatic impairment; } 10 \text { mg dose } \\
\text { recommended for mild-to-moderate } \\
\text { hepatic impairment }\end{array}$ \\
\hline Renal impairment & $\begin{array}{l}\text { Use } 25 \mathrm{mg} \text { starting dose in severe renal } \\
\text { impairment }(\mathrm{CrCl}<30 \mathrm{~mL} / \text { minute })\end{array}$ & $\begin{array}{l}\text { No dosage adjustment needed in mild, } \\
\text { moderate, or severe renal impairment; } \\
\text { however, vardenafil has not been } \\
\text { studied in dialysis patients }\end{array}$ & $\begin{array}{l}\text { Moderate impairment ( } \mathrm{CrCl} 31-50 \\
\mathrm{~mL} / \mathrm{min} \text { ); use starting dose of } 5 \mathrm{mg} \\
\text { not more than once daily, and the } \\
\text { maximum dose should be limited to } \\
10 \mathrm{mg} \text { not more than once every } 48 \\
\text { hours; for patients with severe renal } \\
\text { disease on hemodialysis, the maximum } \\
\text { dose is } 5 \mathrm{mg}\end{array}$ \\
\hline Elderly & $\begin{array}{l}\text { A starting dose of } 25 \mathrm{mg} \text { is recommended } \\
\text { in patients older than } 65 \text { years }\end{array}$ & $\begin{array}{l}\text { A starting dose of } 5 \mathrm{mg} \text { should be con- } \\
\text { sidered in patients } 65 \text { years and older }\end{array}$ & No dosage adjustment needed \\
\hline
\end{tabular}

$\mathrm{CrCl}=$ creatinine clearance; $\mathrm{PDE} 5=$ phosphodiesterase type 5 .

(80 mg) doses produced increases in the QT interval similar to that of $400 \mathrm{mg}$ of moxifloxicin. While the clinical impact of these changes is unknown, the coadministration of vardenafil with Class IA and Class III antiarrhythmic medications should be avoided. Patients with congenital QT prolongation should also avoid vardenafil use..$^{15}$

The contraindications, warnings, and precautions for intracavernosal and transurethral products are exactly the same with one exception. Transurethral alprostadil should not be used for sexual intercourse with a woman who is 
TABLE 16 Indications and Dosage for Alprostadil

\begin{tabular}{|c|c|c|c|}
\hline & $\begin{array}{c}\text { Caverject } \\
\text { (Intracavernous Alprostadil) }\end{array}$ & $\begin{array}{c}\text { Edex } \\
\text { (Intracavernous Alprostadil) }\end{array}$ & $\begin{array}{c}\text { MUSE } \\
\text { (Transurethral Alprostadil) }\end{array}$ \\
\hline FDA indication & $\begin{array}{l}\text { Erectile dysfunction due to neurogenic, } \\
\text { vasculogenic, psychogenic, or mixed } \\
\text { etiology; intracavernous alprostadil is } \\
\text { also indicated as an adjunct to other } \\
\text { diagnostic tests in the diagnosis of } \\
\text { erectile dysfunction. }\end{array}$ & $\begin{array}{l}\text { Intracavernous alprostadil is indicated } \\
\text { for the treatment of erectile dysfunc- } \\
\text { tion due to neurogenic, vasculogenic, } \\
\text { psychogenic, or mixed etiology. }\end{array}$ & $\begin{array}{l}\text { Transurethral alprostadil is indicated for } \\
\text { the treatment of erectile dysfunction. }\end{array}$ \\
\hline \multirow[t]{3}{*}{ Usual adult dose } & $\begin{array}{l}\text { The dose of intracavernous alprostadil } \\
\text { should be individualized for each } \\
\text { patient by careful titration under } \\
\text { supervision by the physician. Dosage } \\
\text { should be initiated at } 2.5 \mathrm{mcg} \text { and } \\
\text { gradually titrated upward according to } \\
\text { response and erection duration. For } \\
\text { spinal cord injury patients, lower initial } \\
\text { doses of } 1.25 \text { mg are recommended. No } \\
\text { more than } 2 \text { doses during an initial } \\
\text { titration should be given in } 24 \text { hours. }\end{array}$ & $\begin{array}{l}\text { The dose of intracavernous alprostadil } \\
\text { should be individualized for each } \\
\text { patient by careful titration under } \\
\text { supervision by the physician. Dosage } \\
\text { should be initiated at } 2.5 \mathrm{mcg} \text { and } \\
\text { gradually titrated upward according to } \\
\text { response and erection duration. For } \\
\text { spinal cord injury patients, lower initial } \\
\text { doses of } 1.25 \text { mg are recommended. No } \\
\text { more than } 2 \text { doses during an initial } \\
\text { titration should be given in } 24 \text { hours. }\end{array}$ & $\begin{array}{l}\text { The dose of transurethral alprostadil } \\
\text { should be individualized for each } \\
\text { patient by careful titration under } \\
\text { supervision by the physician. Dosage } \\
\text { should be initiated at } 125 \text { to } 250 \text { mcg } \\
\text { and gradually titrated upward in a } \\
\text { stepwise manner until the patient } \\
\text { achieves an erection adequate for inter- } \\
\text { course. }\end{array}$ \\
\hline & $\begin{array}{l}\text { Dosage range } 2.5 \text { to } 60 \mathrm{mcg} \text {; mean } \\
\text { dose in clinical studies was } 17.8 \mathrm{mcg} \text {. }\end{array}$ & $\begin{array}{l}\text { Dosage range } 1 \text { to } 40 \text { mcg; mean dose } \\
\text { in clinical studies was } 21.9 \text { mcg. }\end{array}$ & Dosage range 125 to $1,000 \mathrm{mcg}$. \\
\hline & $\begin{array}{l}\text { The recommended frequency is } 3 \text { times } \\
\text { weekly with 24-hour periods between } \\
\text { doses. }\end{array}$ & $\begin{array}{l}\text { The recommended frequency is } 3 \text { times } \\
\text { weekly with } 24 \text { hour periods between } \\
\text { doses. }\end{array}$ & $\begin{array}{l}\text { Most men in clinical studies required } \\
\text { the } 500 \text { or the } 1,000 \text { mcg dose to } \\
\text { achieve an adequate erection. } \\
\text { The maximum frequency is } 2 \text { adminis- } \\
\text { trations within a } 24 \text {-hour period. }\end{array}$ \\
\hline
\end{tabular}

pregnant or could become pregnant, unless the couple uses a condom barrier. This precaution is based on animal data that showed embryotoxic effects when alprostadil was administered as a subcutaneous bolus to pregnant female rats (transurethral alprostadil product information). Table 13 lists the contraindications, warnings, and precautions as stated in intracavernous and transurethral alprostadil product information. ${ }^{11-13}$

\section{Drug/Food Interactions}

Drug and food interactions with PDE5 inhibitors are presented in Table 14

\section{Use in Pregnancy/Nursing}

Transurethral alprostadil should not be used for sexual intercourse with a woman who is pregnant or could become pregnant, unless the couple uses a condom barrier. ${ }^{13}$

Vardenafil, sildenafil, and tadalafil are listed as Pregnancy Category B drugs. While no evidence of fetal or embryonic toxicity was found in animal studies, there are no adequate and well-controlled trials of vardenafil, sildenafil, or tadalafil in pregnant women. ${ }^{14-16}$

In animal studies, tadalafil and vardenafil were secreted into the milk of lactating rats at concentrations 2.4-fold (tadalafil) and 10-fold (vardenafil) greater than found in the plasma. It is not known if these agents are excreted in human breast milk. There is no information on sildenafil and lactation. ${ }^{14-16}$

\section{Indications/Dosing}

The indications, usual adult dose, and dose for special populations for all FDA-approved ERD drugs are listed in Tables 15 and Table 16. ${ }^{11-16}$

\section{Conclusion}

All 3 PDE5 inhibitors have significant efficacy in the treatment of general ERD and ERD associated with diabetes and postprostatectomy. Placebo-controlled trials have also shown sildenafil to have efficacy for patients with ERD associated with depression and spinal cord injury.

There are no head-to-head clinical studies comparing the efficacy and safety of sildenafil with vardenafil or tadalafil. Sildenafil has by far the highest number of controlled studies confirming its safety and efficacy and is recommended as first-line ERD therapy when a nonspecific therapy is appropriate. The PDE5 inhibitors differ in their duration of action. Sildenafil and vardenafil seem to have similar duration of action of about 
TABLE 17 Erectile Dysfunction: Clinical Summary Grid

\begin{tabular}{|c|c|c|c|c|c|c|}
\hline Decision Criteria & Sildenafil & Vardenafil & Tadalafil & $\begin{array}{c}\text { Caverject } \\
\text { (Intracavernous } \\
\text { Alprostadil) }\end{array}$ & $\begin{array}{c}\text { Edex } \\
\text { (Intracavernous } \\
\text { Alprostadil) }\end{array}$ & $\begin{array}{c}\text { MUSE } \\
\text { (Transurethral } \\
\text { Alprostadil) }\end{array}$ \\
\hline $\begin{array}{l}\text { Effectiveness } \\
\text { outcomes }\end{array}$ & $\begin{array}{l}\text { Refill rate lower than } \\
\text { would be expected } \\
\text { from controlled clini- } \\
\text { cal studies; real-world } \\
\text { success rate optimized } \\
\text { by education, follow } \\
\text { up, and management } \\
\text { of ERD risk factors }\end{array}$ & None & None & None & None & None \\
\hline \multirow{4}{*}{$\begin{array}{l}\text { Efficacy outcomes: } \\
\text { POEM }\end{array}$} & Onset $30-40$ minutes & Onset 30-40 minutes & Onset 16-40 minutes & Onset 5-20 minutes & Onset 5-20 minutes & Onset 5-10 minutes \\
\hline & Duration 4 hours & Duration 4 hours & $\begin{array}{l}\text { Duration up to } 36 \\
\text { hours }\end{array}$ & $\begin{array}{l}\text { Long duration of } \\
\text { erection }\end{array}$ & $\begin{array}{l}\text { Long duration of } \\
\text { erection }\end{array}$ & $\begin{array}{l}\text { Offers an alternative if } \\
\text { first-line agents fail or } \\
\text { are contraindicated }\end{array}$ \\
\hline & $\begin{array}{l}57 \% \text { successful } \\
\text { intercourse }\end{array}$ & $\begin{array}{l}50 \%-65 \% \text { successful } \\
\text { intercourse }\end{array}$ & $\begin{array}{l}53 \%-70 \% \text { successful } \\
\text { intercourse }\end{array}$ & $\begin{array}{l}\text { Offers an alternative if } \\
\text { first-line agents fail or } \\
\text { are contraindicated }\end{array}$ & $\begin{array}{l}\text { Offers an alternative if } \\
\text { first-line agents fail or } \\
\text { are contraindicated }\end{array}$ & $\begin{array}{l}\text { Lower efficacy rate than } \\
\text { injectable products. }\end{array}$ \\
\hline & $\begin{array}{l}\text { Improved quality of } \\
\text { life }\end{array}$ & $\begin{array}{l}\text { Efficacy in sildenafil } \\
\text { nonresponders }\end{array}$ & Enhanced spontaneity & $\begin{array}{l}85 \%-90 \% \text { erections } \\
\text { adequate for inter- } \\
\text { course }\end{array}$ & $\begin{array}{l}85 \%-90 \% \text { erections } \\
\text { adequate for inter- } \\
\text { course }\end{array}$ & \\
\hline DOE & $\begin{array}{l}\text { Most extensive efficacy } \\
\text { data in widest patient } \\
\text { population of all ERD } \\
\text { agents } \\
\text { Improved IIEF scores } \\
\text { and other ERD meas- } \\
\text { ures compared with } \\
\text { placebo }\end{array}$ & $\begin{array}{l}\text { Improved IIEF scores } \\
\text { and other ERD meas- } \\
\text { ures compared with } \\
\text { placebo }\end{array}$ & $\begin{array}{l}\text { Improved IIEF scores } \\
\text { and other ERD meas- } \\
\text { ures compared with } \\
\text { placebo }\end{array}$ & $\begin{array}{l}\text { Improved erectile } \\
\text { assessment scale } \\
\text { scores }\end{array}$ & $\begin{array}{l}\text { Improved erectile } \\
\text { assessment scale } \\
\text { scores }\end{array}$ & $\begin{array}{l}\text { Improved erectile } \\
\text { assessment scale } \\
\text { scores }\end{array}$ \\
\hline Safety & $\begin{array}{l}\text { Headaches, flushing, } \\
\text { rhinitis, dyspepsia } \\
\text { Blue vision color } \\
\text { change 3\% } \\
\text { Most extensive safety } \\
\text { data }\end{array}$ & $\begin{array}{l}\text { Headaches, flushing, } \\
\text { rhinitis, dyspepsia } \\
\text { Infrequent reports of } \\
\text { visual changes }<2 \% \\
\text { Prolongs QT interval }\end{array}$ & $\begin{array}{l}\text { Headaches, flushing, } \\
\text { rhinitis, dyspepsia } \\
\text { Rare reports of visual } \\
\text { changes } \\
\text { More frequent myal- } \\
\text { gias and back pain }\end{array}$ & $\begin{array}{l}\text { Injection site } \\
\text { hematoma, ecchy- } \\
\text { mosis, edema, penile } \\
\text { fibrosis risk, penile } \\
\text { pain, prolonged } \\
\text { erection, and } \\
\text { hypotension }\end{array}$ & $\begin{array}{l}\text { Injection site } \\
\text { hematoma, ecchy- } \\
\text { mosis, edema, penile } \\
\text { fibrosis risk, } \\
\text { hypotension, penile } \\
\text { pain, and prolonged } \\
\text { erection }\end{array}$ & $\begin{array}{l}\text { Penile pain,urethral } \\
\text { bleeding, burning, } \\
\text { testicular pain, and } \\
\text { hypotension } \\
\text { Low rate of prolonged } \\
\text { erection and priapism }\end{array}$ \\
\hline Clinical attributes & $\begin{array}{l}\text { Ease of use } \\
\text { Recommended as } \\
\text { first-line in ERD } \\
\text { guidelines }\end{array}$ & Ease of use & $\begin{array}{l}\text { Ease of use } \\
\text { Less frequent } \\
\text { administration } \\
\text { Pharmacokinetics } \\
\text { not affected by a } \\
\text { high-fat meal }\end{array}$ & $\begin{array}{l}\text { Requires initial } \\
\text { titration in } \\
\text { physician office } \\
\text { Limited to } 3 \text { times } \\
\text { a week } \\
\text { Penile injections } \\
\text { necessary }\end{array}$ & $\begin{array}{l}\text { Requires initial } \\
\text { titration in } \\
\text { physician office } \\
\text { Limited to } 3 \text { times } \\
\text { a week } \\
\text { Penile injections } \\
\text { necessary }\end{array}$ & $\begin{array}{l}\text { Requires initial } \\
\text { titration in } \\
\text { physician office } \\
\text { Can be used } \\
\text { twice in } 24 \text { hours } \\
\text { No needles or } \\
\text { syringes to } \\
\text { dispose of or } \\
\text { transport }\end{array}$ \\
\hline
\end{tabular}

4 hours, while tadalafil has a duration of action of up to 36 hours. This prolonged duration of action may be a significant advantage for tadalafil since it could allow for increased sexual spontaneity. However, from a side-effect standpoint, it may not be an advantage to have prolonged levels of tadalafil in the systemic circulation.
Tadalafil, sildenafil, and vardenafil have similar common and nonserious adverse events. Yet, tadalafil does have a higher rate of myalgias and back pain that can take several hours to resolve. Vardenafil and especially tadalafil seem to have less propensity for visual changes. However, vardenafil does produce changes in cardiac conduction at therapeutic doses. 
TABLE 18 Outcome Terms in Evidence-Based Medicine

\begin{tabular}{l|l}
\hline Term & Definition \\
\hline Disease-oriented evidence (DOE) & $\begin{array}{l}\text { Refers to surrogate markers associated } \\
\text { with a specific-disease state such as } \\
\text { blood pressure reduction or glucose } \\
\text { and cholesterol lowering }\end{array}$ \\
\hline $\begin{array}{l}\text { Patient-oriented evidence } \\
\text { that matters (POEM) }\end{array}$ & $\begin{array}{l}\text { Refers to clinical events associated with } \\
\text { a disease such as myocardial infarction, } \\
\text { stroke, and death }\end{array}$ \\
\hline Effectiveness & $\begin{array}{l}\text { Evaluation of beneficial effects of a } \\
\text { treatment when assessed under the } \\
\text { usual conditions of clinical practice; } \\
\text { also referred to as efficacy measured in } \\
\text { a real-world setting }\end{array}$ \\
\hline
\end{tabular}

\begin{tabular}{|c|c|}
\hline Agent & $\begin{array}{l}\text { Cost per Average } \\
\text { or Usual Dose }(\$)^{*}\end{array}$ \\
\hline Intracavernous alprostadil injection (Caverject) & 29.50 \\
\hline Intracavernous alprostadil injection (Edex) & 28.00 \\
\hline Transurethral alprostadil insert (MUSE) & 22.00 \\
\hline Sildenafil (Viagra) & 9.09 \\
\hline Vardenafil (Levitra) & 9.20 \\
\hline Tadalafil (Cialis) & 8.90 \\
\hline
\end{tabular}

* Cost obtained from www.drugstore.com on December 26, 2004, for a single dose, based on the average doses used in clinical studies or the usual dose in the package insert.

Note: In actual deliberations, the PET committee is provided with the WellPoint Pharmacy Management national net cost per claim for the most recent calendar quarter available.

This could be especially significant if vardenafil is coadministered with CYP3A4 inhibitors because these drugs interfere with vardenafil metabolism.

Injectable or transurethral alprostadil remains recommended second-line therapy if first-line therapy is ineffective or contraindicated. Injectable alprostadil results in a quicker onset and a higher success rate than transurethral alprostadil, but it may also have a higher rate of prolonged erections or priapism.

Table 17 contains the clinical summary grid that compares and contrasts effectiveness, efficacy, safety, and clinical attributes of the 6 products currently used for the treatment of ERD. Table 18 lists definitions of some of the outcomes terms used in the clinical summary grid. Table 19 contains the comparative costs for a single dose of the ERD agents discussed in this study.

\section{DISCLOSURES}

No outside funding supported this study. The author discloses no potential bias or conflict of interest relating to this article.

\section{REFERENCES}

1. Gray A, Feldman A, McKinlay JB, Longcope C. Age, disease, and changing sex hormone levels in middle-aged men: results of the Massachusetts Male Aging Study. J Clin Endocrinol Metab. 1991;73:1016-25.

2. Feldman HA, Goldstein I, Hatzichristou DG, et al. Impotence and its medical and psychosocial correlates: results of the Massachusetts Male Aging Study. J Urol. 1994;151:54-61.

3. AACE Male Sexual Dysfunction Task Force. American Association of Clinical Endocrinologists medical guidelines for clinical practice for the evaluation and treatment of male sexual dysfunction: a couple's problem-2003 update. Endocr Pract. 2003;9(1):77.

4. European Association of Urology. Guidelines on erectile dysfunction, update February 2002. Available at: www.uroweb.nl/files/uploaded_files/ guidelines/updateEdysfunction.pdf. Accessed January 31, 2004

5. Kirby RS. Impotence: diagnosis and management of male erectile dysfunction. BMJ. 1994;308:957-62.

6. Garcia-Reboll 1, Mulhall FP, Goldstein I. Drugs for the treatment of impotence. Drugs Aging. 1997;2:140-51.

7. DePalma RG. New development in the diagnosis and treatment of impotence. West J Med. 1996;164(1):55-61.

8. Godschalk MF, Sison A, Mulligan T. Management of erectile dysfunction by the geriatrics. J Am Geriatr Soc. 1997;45:1240-46.

9. NIH Consensus Conference. Impotence. NIH Consensus Development Panel on Impotence. JAMA. 1993;270(1):83-90.

10. Seftel AD. From aspiration to achievement: assessment and noninvasive treatment of erectile dysfunction in aging men. J Am Geriatr Soc. 2005;53: 119-30.

11. Caverject Impulse [product information]. Kalamazoo, MI: Pharmacia and Upjohn; 2003.

12. Edex [product information]. Milwaukee, WI: Schwarz Pharma, Inc.; 2004

13. MUSE [product information]. Mountain View, CA: Vivus; 2003.

14. Viagra [product information]. New York, NY: Pfizer Labs; 2003.

15. Levitra [product information]. West Haven, CT: Bayer Health Care; 2004

16. Cialis [product information]. Indianapolis,IN: Lilly ICOS; 2005.

17. Keating GM, Scott LJ. Vardenafil: a review of its use in erectile dysfunction. Drugs. 2003;63:2673-2703.

18. Corbin JD, Frances SH. Pharmacology of phosphodiesterase-5 inhibitors. Int J Clin Pract. 2002;56(6):453-59.

19. Hellstrom WJG, Overstreet JW, Yu A, et al. Tadalafil has no detrimental effect on human spermatogenesis or reproductive hormones. J Urol. 2003;170: 887-91.

20. Emmick JT, Stuewe SR, Mitchell M. Overview of the cardiovascular effects of tadalafil. Eur Heart J. 2002;4(suppl H):H32-H47.

21. Zussman RM, Morales A, Glassner DB, et al. Overall cardiovascular profile of sildenafil citrate. Am J Cardiol. 1999;83(5A):35C-44C.

22. Kloner RA. Cardiovascular effects of the 3 phosphodiesterase- 5 inhibitors approved for the treatment of erectile dysfunction. Circulation. 2004;110:3149-55.

23. Thadani U, Smith W, Nash S, et al. The effect of vardenafil, a potent and highly selective phosphodiesterase inhibitor, for the treatment of erectile dysfunction on the cardiovascular response to exercise in patients with coronary artery disease. J Am Coll Cardiol. 2002;40(11):2006-12.

24. Arruda-Olson AM, Mahoney DW, Nehra A, et al. Cardiovascular effects of sildenafil during exercise in men with known or probable coronary artery disease: a randomized crossover trial. JAMA. 2002;287(6):719-25. 
25. Stark S, Sachse R, Liedl T, et al. Vardenafil increases penile rigidity and tumescence in men with erectile dysfunction after a single oral dose. Eur Urol. 2001;40(2):181-88.

26. Klotz T, Sasche R, Heidrich A, et al. Vardenafil increases penile rigidity and tumescence in erectile dysfunction patients: a RigiScan and pharmacokinetic study. World J Urol. 2001;19:32-39.

27. Eardley I, Ellis P, Boolell M, Wuff M. Onset and duration of action of sildenafil citrate. B J Pharmacol. 2002;53:21S-30S

28. Padma-Nathan H, Rosen RC, Shabsigh R, et al. Cialis (IC351) provides prompt response and extended period of responsiveness for the treatment of men with erectile dysfunction. J Urol. 2001;165(suppl 1):221. Abstract 923.

29. Lea AP, Bryson HM, Balfour JA. Intracavernous alprostadil. A review of its pharmacodynamic and pharmacokinetic properties and therapeutic potential in erectile dysfunction. Drugs Aging. 1996;8(1):56-74.

30. Muhall JP. Deciphering erectile dysfunction drug trials. J Urol. 2003;170: 353-58.

31. Fink HA, Mac Donald R, Rutks IR, et al. Sildenafil for male erectile dysfunction: a systematic review and meta-analysis. Arch Intern Med. 2002;162:1349-60.

32. Hellstrom WJG, Gittelman M, Karlin G, et al. Vardenafil for treatment of men with erectile dysfunction: efficacy and safety in a randomized, doubleblind, placebo-controlled trial. J Andrology. 2002;23(6):763-71.

33. Porst H, Young JM, Schmidt JB, and the International Vardenafil Study Group. Efficacy and tolerability of vardenafil for treatment of erectile dysfunction in patient subgroups. Urology. 2003;62:519-24.

34. Brock GB, McMahon CG, Chen KK, et al. Efficacy and safety of tadalafil for the treatment of erectile dysfunction: results of an integrated analyses of registration trials. J Urol. 2002;168:1332-36.

35. Porst H, Padman-Nathan H, Giuliano F, et al. Efficacy of tadalafil for the treatment of erectile dysfunction at 24 and 36 hours after dosing: a randomized controlled trial. Urology. 2003;62:121-26.

36. Linet OI, Ogrine FG. Efficacy and safety of intracavernosal alprostadil in men with erectile dysfunction. The Alprostadil Study Group. New Engl J Med. 1996;334(14):873-77.

37. Albrecht D, Goldstein I, Kaufman J, et al. Placebo-controlled, doubleblind study of alprostadil alfadex for self-injection therapy at home. J Urol. 1997;157:183.

38. Hellstrom WJG, Bennett AH, Gesundheit N, et al. A double-blind, placebocontrolled evaluation of the erectile response to transurethral alprostadil. Urology. 1996;48(6):851-56.

39. Padma-Nathan H, Hellstrom WJ, Kaiser FE, et al. Medicated Urethral System for Erection (MUSE) Study Group: treatment of men with erectile dysfunction with transurethral alprostadil. New Engl J Med. 1997;336:1-7.

40. Rendell MS, Rajfer J, Wicker PA, Smith MD. Sildenafil for treatment of erectile dysfunction in men with diabetes: a randomized controlled trial. JAMA. 1999;281(5):421-26.

41. Boulton AJ, Selam JL, Sweeney M, Ziegler D. Sildenafil citrate for the treatment of erectile dysfunction in men with type II diabetes mellitus. Diabetologia. 2001;44(10):1296-1301.

42. Stuckey BGA, Jadzinsky MN, Murphy LJ, et al. Sildenafil citrate for treatment of erectile dysfunction in men with type 1 diabetes. Diabetes Care. 2003; 26(2):279-84

43. Goldstein I, Young JM, Fischer J, et al. Vardenafil, a new phosphodiesterase type 5 inhibitor, in the treatment of erectile dysfunction in men with diabetes. Diabetes Care. 2003;26(3):777-83.

44. DeTejada IS, Anglin G, Knight JR, Emmick JT. Effects of tadalafil on erectile dysfunction in men with diabetes. Diabetes Care. 2002;25:2159-64.
45. Zippe CD, Jhaveri FM, Klein EA, et al. Role of Viagra after radical prostatectomy. Urology. 2000;55:241-45.

46. Raina R, Lakin M, Agarwal A, et al. Long-term effect of sildenafil citrate on erectile dysfunction after radical prostatectomy: 3-year follow-up. Urology. 2003;62:110-15.

47. Zagaja GP, Mahoon DA, Aikens JE, et al. Sildenafil in the treatment of erectile dysfunction after radical prostatectomy. Urology. 2000;56:631-34.

48. Brock GB, Nehra A, Lipshultz LI, et al. Safety and efficacy of vardenafil for the treatment of men with erectile dysfunction after radical retropubic prostatectomy. J Urol. 2003;170:1278-83.

49. Data on file. Formulary submission dossier, Eli Lilly and Company; December 2003.

50. Giuliano F, Hulting D, Wagih S, et al. Randomized trial of sildenafil for the treatment of erectile dysfunction in spinal cord injury. Ann Neurol. 1999;46: 15-21.

51. Seidman SN, Roose SP, Menza MA, et al. Treatment of erectile dysfunction in men with depressive symptoms: results of a placebo-controlled trial with sildenafil citrate. Am J Psych. 2001;158:1623-30.

52. Nurnberg GH, Hensley PL, Gelenberg AJ, et al. Treatment of antidepressant associated sexual dysfunction with sildenafil: a randomized controlled trial. JAMA. 2003;289(1):56-64

53. Shokeir AA, Alserafi MA, Mutabagani $\mathrm{H}$. Intracavernosal versus intraurethral alprostadil: a prospective, randomized study. BJU Int. 1999;83(7):812-15

54. Shabsigh R, Padma-Nathan H, Gittleman M, et al. Intracavernous alprostadil alfadex is more efficacious, better tolerated, and preferred over intraurethral alprostadil plus optional actis: a comparative, randomized, crossover, multicenter study. Urology. 2000;55(1):109-13.

55. Engel JD, McVary KT. Transurethral alprostadil as therapy for patients who withdrew or failed prior intracavernous injection therapy. Urology. 1998;51: 687-92.

56. Shabsigh R, Padma-Nathan H, Gittleman M, et al. Intracavernous alprostadil (Edex/Viridal) is effective and safe in patients with erectile dysfunction after failing sildenafil (Viagra). Urology. 2000;55:477-80.

57. Carson C, Hatzichristou DB, Carrier S, et al. Vardenafil exhibits efficacy in men with erectile dysfunction unresponsive to prior sildenafil therapy: results of a phase III clinical trial-Patient Response with Vardenafil in sildenafil nonresponders (PROVEN). Abstract presented at: 5th Annual Fall Research Meeting of the Sexual Medicine Society of North America; October 11, 2003; Denver, $\mathrm{CO}$.

58. Data on file. Pfizer Labs; 2002.

59. El-Galley R, Rutland H, Talic R, et al. Long-term efficacy of sildenafil and tachyphylaxis effect. J Urology. 2001;166:927-31.

60. McCullough AR, Barada JH, Fawzy A, et al. Achieving treatment optimization with sildenafil citrate in patients with erectile dysfunction. Urology. 2002; 60(suppl 2B):28-38.

61. Kloner RA, Mitchell M, Emmick JT. Cardiovascular effects of tadalafil. Am J Cardiol. 2003;92(9A):37M-46M.

62. Mittelman MB, Glassner DB, Orazem J. Clinical trials of sildenafil citrate demonstrate no increase in risk of myocardial infarction and cardiovascular death compared with placebo. Int J Clin Pract. 2003;57(7):597-600.

63. Shakir S, Wilton LV, Boshier A, et al. Cardiovascular events in users of sildenafil: results from first phase of prescription event monitoring in England. BMJ. 2001;322:651-52.

64. Padma-Nathan H, Eardley I, Kloner RA, et al. A 4-year update on the safety of sildenafil citrate. Urology. 2002;60(suppl 2B):67-90. 